\title{
Herschel map of Saturn's stratospheric water, delivered by the plumes of Enceladus ${ }^{\star}$
}

\author{
T. Cavalié ${ }^{1,2}$, V. Hue ${ }^{3}$, P. Hartogh ${ }^{4}$, R. Moreno ${ }^{2}$, E. Lellouch ${ }^{2}$, H. Feuchtgruber ${ }^{5}$, C. Jarchow ${ }^{4}$, T. Cassidy ${ }^{6}$, \\ L. N. Fletcher ${ }^{7}$, F. Billebaud ${ }^{1}$, M. Dobrijevic ${ }^{1}$, L. Rezac ${ }^{4}$, G. S. Orton ${ }^{8}$, M. Rengel ${ }^{4}$, T. Fouchet ${ }^{2}$, and S. Guerlet ${ }^{9}$ \\ ${ }^{1}$ Laboratoire d'Astrophysique de Bordeaux, University of Bordeaux, CNRS, B18N, allée Geoffroy Saint-Hilaire, 33615 Pessac, France \\ e-mail: thibault.cavalie@u-bordeaux. fr \\ ${ }^{2}$ LESIA, Observatoire de Paris, PSL Research University, CNRS, Sorbonne Universités, UPMC University of Paris 06, \\ Université Paris Diderot, Sorbonne Paris Cité, 92195 Meudon, France \\ ${ }^{3}$ Southwest Research Institute, San Antonio, TX 78228, USA \\ ${ }^{4}$ Max-Planck-Institut für Sonnensystemforschung, 37077 Göttingen, Germany \\ ${ }^{5}$ Max Planck Institut für Extraterrestrische Physik, Garching, Germany \\ ${ }^{6}$ Laboratory for Atmospheric and Space Physics, University of Colorado, Boulder, CO 80303, USA \\ ${ }^{7}$ Department of Physics \& Astronomy, University of Leicester, University Road, Leicester LE1 7RH, UK \\ 8 Jet Propulsion Laboratory, California Institute of Technology, 4800 Oak Grove Drive, Pasadena, CA 91109, USA \\ ${ }^{9}$ Sorbonne Universités, UPMC Paris 06, UMR 8539, LMD, 75005 Paris, France
}

Received 24 May 2019 / Accepted 23 July 2019

\begin{abstract}
Context. The origin of water in the stratospheres of giant planets has been an outstanding question ever since its first detection by the Infrared Space Observatory some 20 years ago. Water can originate from interplanetary dust particles, icy rings and satellites, and large comet impacts. Analyses of Herschel Space Observatory observations have proven that the bulk of Jupiter's stratospheric water was delivered by the Shoemaker-Levy 9 impacts in 1994. In 2006, the Cassini mission detected water plumes at the South Pole of Enceladus, which made the moon a serious candidate for Saturn's stratospheric water. Further evidence was found in 2011 when Herschel demonstrated the presence of a water torus at the orbital distance of Enceladus that was fed by the moon's plumes. Finally, water falling from the rings onto Saturn's uppermost atmospheric layers at low latitudes was detected during the final orbits of Cassini's end-of-mission plunge into the atmosphere.

Aims. In this paper, we use Herschel mapping observations of water in Saturn's stratosphere to identify its source.

Methods. We tested several empirical models against the Herschel-HIFI and -PACS observations, which were collected on December 30, 2010, and January 2, 2011, respectively.

Results. We demonstrate that Saturn's stratospheric water is not uniformly mixed as a function of latitude, but peaks at the equator and decreases poleward with a Gaussian distribution. We obtain our best fit with an equatorial mole fraction $1.1 \mathrm{ppb}$ and a half width at half maximum of $25^{\circ}$, when accounting for a temperature increase in the two warm stratospheric vortices produced by Saturn's Great Storm of 2010-2011.
\end{abstract}

Conclusions. This work demonstrates that Enceladus is the main source of Saturn's stratospheric water.

Key words. planets and satellites: individual: Saturn - planets and satellites: individual: Enceladus planets and satellites: atmospheres

\section{Introduction}

The interiors of giant planets are supposedly rich in oxygen (Owen \& Encrenaz 2003; Gautier et al. 2001; Hersant et al. 2004). In the deep hot layers, where thermochemical equilibrium prevails, $\mathrm{H}_{2} \mathrm{O}$ is the most abundant oxygen species. Oxygen species are transported from deep down toward higher levels, but only $\mathrm{CO}$ (and $\mathrm{CO}_{2}$ in Jupiter and Saturn) can reach the stratosphere because of the tropopause cold trap (Lodders \& Fegley 1994; Wang et al. 2015; Cavalié et al. 2017). $\mathrm{H}_{2} \mathrm{O}$ was thus only observed in the tropospheric saturation layers of Jupiter and Saturn (Larson et al. 1975; de Graauw et al. 1997). The detection of oxygen species $\left(\mathrm{H}_{2} \mathrm{O}, \mathrm{CO}\right.$, and $\left.\mathrm{CO}_{2}\right)$ in the stratospheres of the giant planets and Titan (Feuchtgruber et al. 1997; Bézard et al. 2002; Lellouch et al. 2002; Coustenis et al. 1998;

\footnotetext{
${ }^{\star}$ Herschel is an ESA space observatory with science instrument provided by European-led Principal Investigator consortia and with important participation from NASA.
}

Samuelson et al. 1983; Noll et al. 1986; Marten et al. 1993; Burgdorf et al. 2006) thus demonstrated that the upper atmospheres of the giant planets are contaminated by external sources from their close and/or more distant environments.

While oxygen-rich interplanetary dust particles (IDP) produced from asteroid collisions and comet activity are a ubiquitous source (Landgraf et al. 2002; Poppe 2016), these particles seem to be the main $\mathrm{H}_{2} \mathrm{O}$ source only at Uranus and Neptune (Moses \& Poppe 2017) and the emerging overall picture however looks more complex. Other sources can actually be at work such as local sources from planetary icy environments (rings and satellites) (Strobel \& Yung 1979; Connerney 1986; Prangé et al. 2006; Waite et al. 2018; Mitchell et al. 2018; Hsu et al. 2018) or "Shoemaker-Levy 9" (SL9) type cometary impacts (Lellouch et al. 1995). In Jupiter's stratosphere, $\mathrm{H}_{2} \mathrm{O}, \mathrm{CO}$, and $\mathrm{CO}_{2}$ come from SL9 comet fragments (Bézard et al. 2002; Lellouch et al. 2002, 2006; Cavalié et al. 2008a, 2012, 2013). An older cometimpact component was even proposed as the source of the lower 
stratospheric CO (Bézard et al. 2002), even if IDP are another option (Moses \& Poppe 2017). Comets are also the probable source of CO beyond Jupiter (see review in Mandt et al. 2015 and Moses \& Poppe 2017), as seen in Saturn (Cavalié et al. 2009, 2010), Uranus (Cavalié et al. 2014), and Neptune (Lellouch et al. 2005, 2010; Luszcz-Cook \& de Pater 2013; Moreno et al. 2017).

In Saturn, neither the Infrared Space Observatory (ISO) nor the Submillimeter Wave Astronomy Satellite (SWAS) diskaveraged observations (Feuchtgruber et al. 1997; Bergin et al. 2000) had sufficient spectral resolutions or high enough signalto-noise ratios $(\mathrm{S} / \mathrm{N})$ to identify the source of external $\mathrm{H}_{2} \mathrm{O}$. A comet impact is probably not the cause of the observed stratospheric $\mathrm{H}_{2} \mathrm{O}$ (Moses \& Poppe 2017) because of the contradiction between the relatively ancient impacts required to fit the $\mathrm{CO}$ observations of Cavalié et al. (2010) and the relatively short diffusion timescale from the deposition level of cometary material in such impacts (Lellouch et al. 1995; Moreno et al. 2003) down to the $\mathrm{H}_{2} \mathrm{O}$ condensation level (Ollivier et al. 2000; Moses et al. 2000, 2005), which is located between the 1 and a few millibar altitude levels, depending on the latitude. In addition, the $\mathrm{H}_{2} \mathrm{O} / \mathrm{CO}$ in Saturn's stratosphere ( 0.15 - Moses \& Poppe 2017) is too large to be characteristic of a cometary impact that delivers mostly $\mathrm{CO}\left(\mathrm{H}_{2} \mathrm{O} / \mathrm{CO}<0.01\right.$ in Neptune). This tends to indicate a source that is steadier than a discrete comet impact. Previous observations of Saturn with the Herschel-Heterodyne Instrument for the Far Infrared (HIFI) led to the detection of an $\mathrm{H}_{2} \mathrm{O}$ torus at the orbit of Enceladus (Hartogh et al. 2011), which is fed by the plumes of this moon (Hansen et al. 2006; Porco et al. 2006; Waite et al. 2006). The fate of $\mathrm{H}_{2} \mathrm{O}$ from this torus is eventually to spread in Saturn's system (Cassidy \& Johnson 2010) and a fraction is predicted to fall into Saturn's stratosphere with a distribution centered around the equator. Based on these models, and in comparison with the Herschel observations, Hartogh et al. (2011) tentatively concluded that Enceladus was the source of Saturn's stratospheric water. It should be noted that a fraction is also expected to feed Titan's atmosphere as well, and models show that the flux expected at Titan and originating from the Enceladus water torus could explain the observations (Moreno et al. 2012; Lara et al. 2014; Dobrijevic et al. 2014; Rengel et al. 2014; Hickson et al. 2014).

Recent in situ measurements of Cassini during the proximal orbits of its end-of-mission demonstrated the existence of a flux of material from the rings to Saturn's atmosphere. The Magnetospheric Imaging Instrument (MIMI), the Ion and Neutral Mass Spectrometer (INMS), and the Cosmic Dust Analyzer (CDA) instruments have measured an infall of material originating from the D-ring: (i) neutral icy grains within $2^{\circ}$ around the equator, (ii) various gases including $\mathrm{H}_{2} \mathrm{O}$ (concentrated at the $24 \%$ level) in a latitudinal band of $8^{\circ}$ centered on the equator (Waite et al. 2018), (iii) and charged grains transported to higher latitudes along the magnetic field lines (Mitchell et al. 2018; Hsu et al. 2018). This latter phenomenon is the long anticipated ring rain phenomenon (Connerney \& Waite 1984; Connerney 1986; Prangé et al. 2006; Moore et al. 2010, 2015; O’Donoghue et al. 2017). The mass flux of infalling dust and gas are estimated to be $\sim 5 \mathrm{~kg} \mathrm{~s}^{-1}$ (Mitchell et al. 2018) and $\sim 10^{4} \mathrm{~kg} \mathrm{~s}^{-1}$ (Waite et al. 2018; Perry et al. 2018), respectively. The sources that can explain the presence of $\mathrm{H}_{2} \mathrm{O}$ in Saturn's stratosphere are thus in principle IDP, Enceladus plumes, and Saturn's rings.

Disentangling the various sources of externally supplied water in giant planet stratospheres, and thus in Saturn, was a key objective of the Herschel key program Herschel Solar System Observations (HssO) (Hartogh et al. 2009). In Sect. 2 of this paper, we present the first disk-resolved mapping observations of $\mathrm{H}_{2} \mathrm{O}$ in Saturn's stratosphere and a disk-averaged observation obtained with the Photodetector Array Camera and Spectrometer (PACS) (Poglitsch et al. 2010) and HIFI (de Graauw et al. 2010), respectively; these two instruments were on board the ESA Herschel Space Observatory (Pilbratt et al. 2010). Using a combination of models presented in Sect. 3, we derive the $\mathrm{H}_{2} \mathrm{O}$ meridional distribution in Saturn's stratosphere in Sect. 4. We discuss its origin according to our results in Sect. 5 and give our conclusion in Sect. 6.

\section{Observations}

In Table 1, we present a summary of the observations of Saturn's stratospheric $\mathrm{H}_{2} \mathrm{O}$ performed with Herschel-PACS and Herschel-HIFI. We also add the relevant geometry of Saturn, as obtained from the Jet Propulsion Laboratory/Horizons database. In the following sections, we detail the observations and the data reduction.

\subsection{PACS observations}

We observed Saturn with Herschel-PACS in the framework of the HssO Key Program on January 2, 2011 with the aim of mapping the distribution of $\mathrm{H}_{2} \mathrm{O}$ in the stratosphere of the planet. We used PACS in its line spectrometry mode (Poglitsch et al. 2010). The integral field spectrometer consists of $5 \times 5$ spatial pixels $\left(\sim 50^{\prime \prime} \times 50^{\prime \prime}\right.$ on sky), each covering a short instantaneous wavelength range sampled by 16 spectral pixels. Our observation of Saturn consists of a $3 \times 3$ raster map with $3^{\prime \prime}$ separation at $66.44 \mu \mathrm{m}$. We thus pointed the 25 pixel array over nine different positions to record an oversampled 225 point map of Saturn at $66.44 \mu \mathrm{m}$. For the observation of such an intense farinfrared continuum source, we had to use a nonstandard mode, in which the spectrometer readout electronics were configured to the shortest possible reset intervals of $1 / 32 \mathrm{~s}$, to avoid detector saturation. The half-power beam width (HPBW) of Herschel is 9.4" for the PACS observations, which results in covering latitudes from $-23^{\circ}$ to $45^{\circ}$ for a beam centered on the planet. The spectral resolving power is $\sim 2500-3000$.

We ran the basic data reduction with the Herschel Interactive Processing Environment 8.0 (HIPE; Ott 2010) and performed additional processing (flat-fielding, outlier removal, and rebinning) with standard Interactive Data Language (IDL) tools. An example of raw spectra for one of the raster positions of the map in presented in Fig. 1. The 225 spectra corresponding to all raster positions of the map can be found in Fig. A.1. After subtracting the coordinates of Saturn given by the JPL/Horizon ephemeris, we found from the continuum distribution that the map has a residual pointing offset of $2.5^{\prime \prime}$ in RA and $0.3^{\prime \prime}$ in dec. The position of the line peak presents some scatter, which results from a combination of two effects: a Doppler shift caused by the rapid rotation of the planet and wavelength shifts induced by the nonuniform illumination of the instrument slit on some of the raster positions (Poglitsch et al. 2010). A baseline, caused by standing waves excited by the strong continuum emission of the planet in the instrument, was then removed from the raw spectra by fitting a polynomial. This step introduces on average a 10 $15 \%$ uncertainty on the line amplitude. The $\mathrm{H}_{2} \mathrm{O}$ line is detected in all positions within the disk and on positions within $\sim$ one beam from the planetary limb. For the reasons detailed in Cavalié et al. (2013), reasonable uncertainties cannot be achieved on an absolute flux calibration and we have to express the spectra in terms of line-to-continuum ratio $(l / c)$ to cancel out the absolute response. In addition, the line width is purely instrumental (and 
Table 1. Summary of the Herschel-PACS and Herschel-HIFI observations of Saturn and the relevant observation geometry.

\begin{tabular}{llll}
\hline \hline Start date & $2010-12-3103: 33: 17$ & Date for computations & $2010-12-3103: 40: 00$ \\
OD & 596 & Ang. Diam. & $17.20^{\prime \prime}$ \\
Obs. ID & 1342212192 & $\lambda_{\text {obs }}$ & $226.63^{\circ}$ \\
$\Delta t(\mathrm{~s})$ & 744 & $\phi_{\text {obs }}$ & $12.42^{\circ}$ \\
Instrument & HIFI & $\lambda_{\odot}$ & $231.99^{\circ}$ \\
Frequency & $1097.365 \mathrm{GHz}$ & $\phi_{\odot}$ & $9.28^{\circ}$ \\
& & $a_{\mathrm{NP}}$ & $357.29^{\circ}$ \\
\hline Start date & $2011-01-0210: 32: 21$ & Date for computations & $2011-01-0210: 47: 00$ \\
OD & 598 & Ang. Diam. & $17.26^{\prime \prime}$ \\
Obs. ID & 1342212275 & $\lambda_{\text {obs }}$ & $288.72^{\circ}$ \\
$\Delta t(\mathrm{~s})$ & 1693 & $\phi_{\mathrm{obs}}$ & $12.46^{\circ}$ \\
Instrument & $\mathrm{PACS}$ & $\lambda_{\odot}$ & $294.11^{\circ}$ \\
Wavelength & $66.44 \mu \mathrm{m}$ & $\phi_{\odot}$ & $9.32^{\circ}$ \\
& & $a_{\mathrm{NP}}$ & $357.30^{\circ}$ \\
\hline
\end{tabular}

Notes. OD means Herschel operational day, $\Delta t$ is the total integration time. Ang. Diam. is Saturn's equatorial angular diameter, $\lambda_{\mathrm{obs}}$ and $\phi_{\mathrm{obs}}$ are the longitude and latitude (respectively) of the sub-observer point, $\lambda_{\odot}$ and $\phi_{\odot}$ are the longitude and latitude, respectively, of the subsolar point, $a_{\mathrm{NP}}$ is the north polar angle. The solar longitude of Saturn $\left(L_{\mathrm{S}}\right)$ is $17^{\circ}$. All latitudes in this table are planetographic and all longitudes are System III west longitudes. The physical parameters of Saturn (right column) were obtained from the JPL/Horizons database.

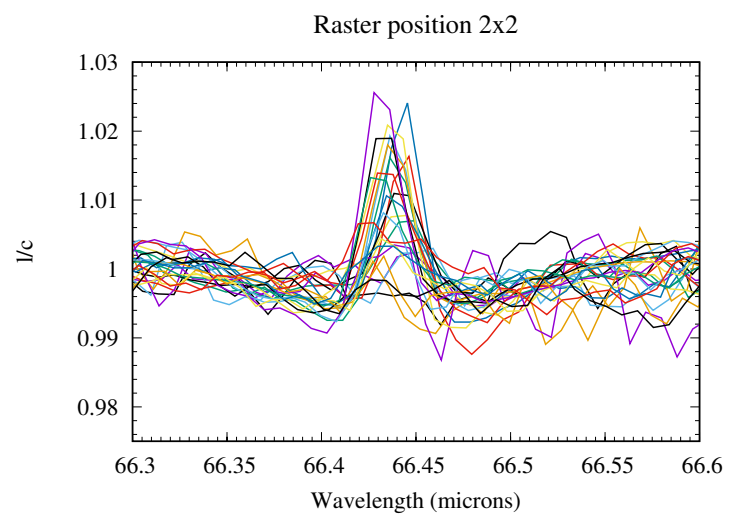

Fig. 1. Twenty-five raw spectra obtained with the PACS $5 \times 5$ detector array and extracted from one of the $3 \times 3$ raster map positions on Saturn at $66.44 \mu \mathrm{m}$. The $\mathrm{H}_{2} \mathrm{O}$ line is detected in all spectra corresponding to pointings on the planetary disk or close to the planetary limb. Spectra without an $\mathrm{H}_{2} \mathrm{O}$ line correspond to pointings far off the planetary limb. Before fitting the lines with a Gaussian to compute their area, a polynomial baseline was removed from these spectra. The line peak $\mathrm{S} / \mathrm{N}$ around the limb is $\sim 15-45$.

Gaussian) and can vary from one position to another because of varying beam filling factors on the detector array. Therefore, the map must be analyzed in terms of line area. We fit the lines with a Gaussian and computed their area from the Gaussian fit parameters. According to the noise on the line peak intensity and line width, we estimated that the line area map can be safely analyzed for positions within the planetary disk or in the vicinity of the limb (see Fig. 2). We found a $1 \sigma$ rms of 0.0023 (in units of microns $\times \%$ of the continuum) when adding quadratically the baseline removal uncertainty and the spectral noise. We used this value in our $\chi^{2} / N$ calculations ( $N$ is the number of pixels in the map). The resulting continuum and line-area maps are presented in Fig. 2. The average $\mathrm{S} / \mathrm{N}$ around the limb, i.e., where the lines have the biggest contrast ${ }^{1}$, is $\sim 15-45$ on the line area. There is enhanced emission at low latitudes with respect to mid

1 At a given latitude, the increased emission at the limb (compared to the disk center) is a geometrical effect caused by limb brightening in the line and limb darkening in the continuum. to high latitudes, and a local minimum around the north pole. This enables us to demonstrate the difference in water emission between the poles and equatorial region despite the limited spatial resolution.

\subsection{HIFI observations}

In addition, and in an attempt to check the PACS results at the planetary scale, we used the disk-averaged observation of Saturn conducted with the HIFI instrument (de Graauw et al. 2010) at $1097 \mathrm{GHz}$ on December 31, 2010 using a dual beamswitch mode (Roelfsema et al. 2012), which facilitates pointing to the deep sky for calibration by moving the secondary mirror instead of the primary. We chose this strong $\mathrm{H}_{2} \mathrm{O}$ line because it is not affected by Enceladus torus absorption. This absorption is seen only in lines, such as those at 557 and $1113 \mathrm{GHz}$ (Hartogh et al. 2011), which have a lower state energy relative to the ground state $\left(23.7944\right.$ and $0.0 \mathrm{~cm}^{-1}$, respectively); the lower state energy of the $1097 \mathrm{GHz}$ line is $136.7617 \mathrm{~cm}^{-1}$. Given that the Herschel HPBW is 19.3" (i.e., bigger than Saturn) and the spectral resolution of the Wide Band Spectrometer (WBS) is $1.1 \mathrm{MHz}$, the line appears smeared because of the fast rotation of Saturn $\left(9.9 \mathrm{~km} \mathrm{~s}^{-1}\right.$ at the equator). We processed the data with the standard HIPE 8.2.0 pipeline (Ott 2010) up to level 2 for the $H$ and $V$ polarizations. We then removed standing waves using a Lomb (1976) algorithm before averaging the two polarizations and smoothed the spectral resolution to $10 \mathrm{MHz}$. This last operation barely changes the line shape as the width of the line is already $\sim 35 \mathrm{MHz}$ because of the aforementioned rotational smearing. The resulting line is shown in Fig. 3 and we note a small asymmetry and the fact that the line-center frequency is not aligned with the line rest frequency. This asymmetry is caused by a pointing offset of $1.5^{\prime \prime}$ in the planetary western limb direction, which we account for in what follows. We performed no absolute calibration and thus cannot constrain the north-south pointing offset, which must be on the order of a few arcseconds according to the observatory pointing uncertainty at this frequency (Roelfsema et al. 2012). We accounted for the double sideband (DSB) response of the instrument by assuming a normalized sideband ratio $G_{\mathrm{USB}}(H+V)=0.469 \pm 0.016$ (Higgins et al. 2014) and a ratio of the continuum in the upper to lower sidebands of 1.037 (according to our continuum model) and 

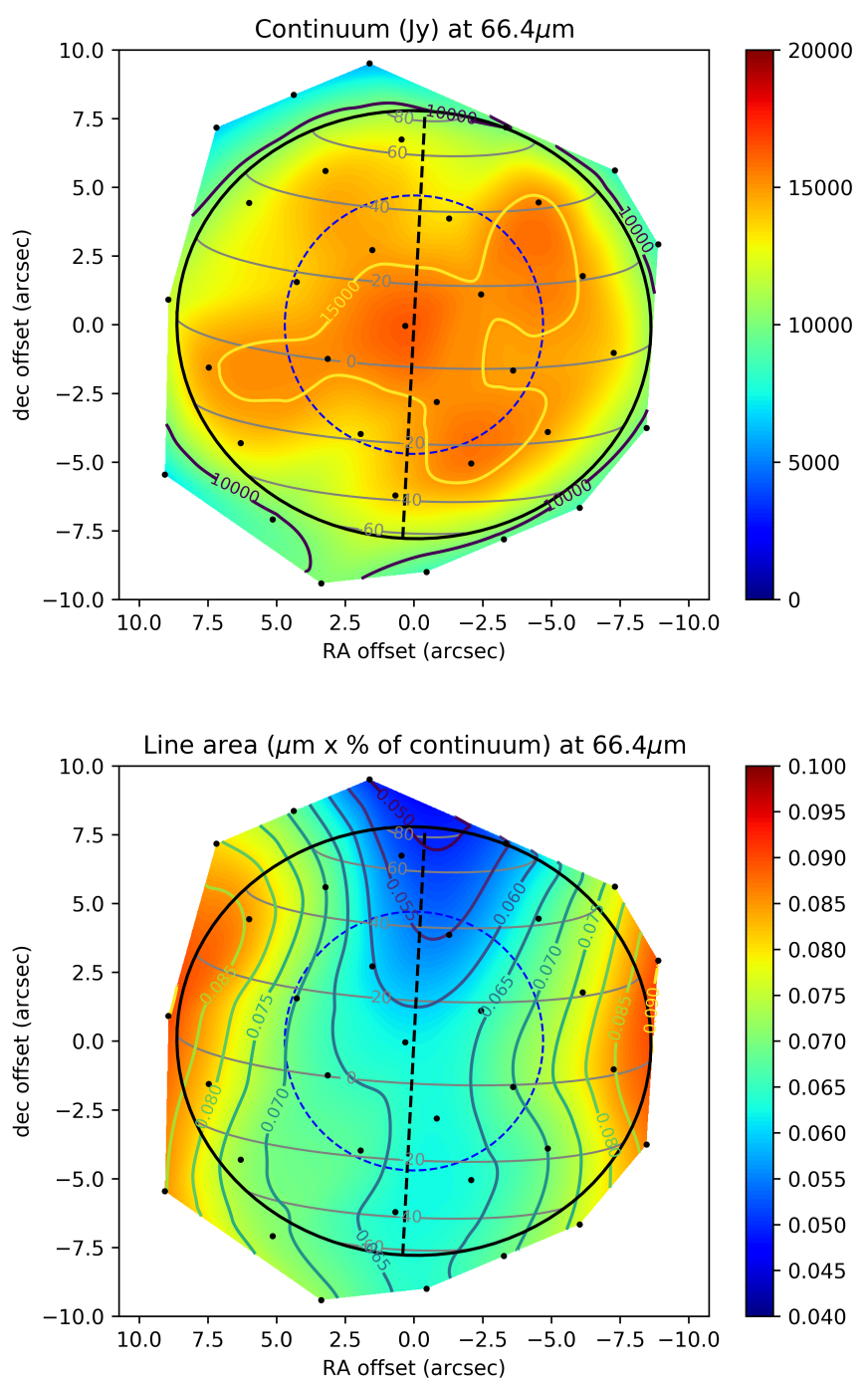

Fig. 2. Water map at $66.44 \mu \mathrm{m}$ observed by the PACS spectrometer on January 2, 2011. Saturn is represented by the black ellipse and its rotation axis is shown with a black dashed line. Iso-latitudes are plotted with gray lines. The beam is represented by a blue dashed circle. Each black dot represents the central position of a pixel of the raster map. Top: image of the continuum (in Jy), after the residual pointing offset was corrected. Bottom: the line area in microns $\times \%$ of the continuum is shown.

produced a single sideband (SSB) spectrum that we used for our analyses in this paper in terms of $l / c$. The total uncertainty on the $l / c$, when accounting for the uncertainties caused by the normalized sideband ratio, baseline removal process, and spectral noise, is $3 \%$, i.e., $0.08 \mathrm{~K}$. All our $\chi^{2} / N$ calculations are based on this rms value, which does not include the absolute calibration uncertainties that are estimated to be $5 \%$.

\section{Modeling}

In this section, we present how we modeled the Herschel observations. We first detail how we built a temperature field representative of the epoch of our observations. Then, we describe the radiative-transfer model we applied in our data analysis.

\subsection{Thermal model}

The pressure-latitude thermal field we used in our radiativetransfer modeling was extracted from the spline interpolation

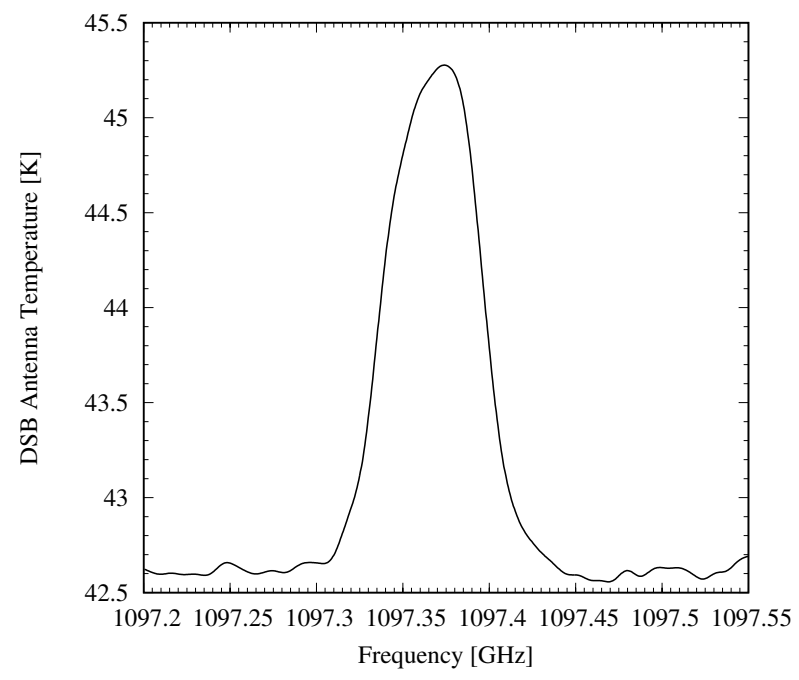

Fig. 3. Water line at $1097.365 \mathrm{GHz}$ observed by the HIFI spectrometer on December 31, 2010. The spectrum is expressed in terms of kelvins on the DSB scale. The asymmetry seen in the line shape is caused by a pointing offset of $1.5^{\prime \prime}$ in the planet western limb direction. The northsouth pointing offset is on the order of a few arcseconds, but cannot be constrained further because of calibration uncertainties. The total uncertainty on the $l / c$ is $3 \%$.

made over time to fill in missing parts of Cassini's Composite Infrared Spectrometer (CIRS) time series spanning the full mission (Fletcher et al. 2017). We could thus extract the zonally averaged stratospheric temperatures pertinent to the dates of the Herschel observations. At the time of our observations, the CIRS dataset featured significant temperature anomalies associated with the planetary storm that erupted at the end of 2010 (Sánchez-Lavega et al. 2011; Fischer et al. 2011; Fletcher et al. 2011). This storm significantly changed the northern stratospheric temperatures in a $20^{\circ}$ latitude band centered on $40^{\circ} \mathrm{N}$ planetocentric. Two hot stratospheric vortices formed well above the tropospheric storm and were nicknamed "beacons" because of their bright appearance in the infrared and the rapid rotation of Saturn. These beacons eventually merged in April-May 2011 to form a huge vortex in which a temperature increase of up to $80 \mathrm{~K}$ was measured at the 2 mbar level. But, because our observations occurred very early in the time evolution of the storm and beacons, we first chose to produce a thermal field representative of storm-free conditions by removing any coverage between $20 \mathrm{~N}$ and $60 \mathrm{~N}$ and 2011-2012 in the spline smoothing; we include these beacons later in the paper (in Sect. 4.3). We took the thermal field produced for the date closest to our observations, i.e., December 28, 2010. The stratospheric temperatures in the 0.110 mbar range, i.e., roughly the range in which the HIFI and PACS lines are sensitive, are quite symmetric in latitude with respect to the equator up to $\sim 40^{\circ}$, with a gradient from $40 \mathrm{~S}$ to the south pole $<5 \mathrm{~K}$ and a $-10 \mathrm{~K}$ gradient from $40 \mathrm{~N}$ to the north pole, as already hinted for observations at similar $L_{\mathrm{S}}$ by Sinclair et al. (2013). The CIRS observations used $600-1400 \mathrm{~cm}^{-1}$ in nadir geometry, therefore probing only the $0.2-5$ mbar and 70 250 mbar ranges. Outside of these ranges, the profile goes back to an a priori profile built by averaging Guerlet et al. (2009, 2010) limb observations, which probed higher stratospheric altitudes than the study of Fletcher et al. (2017), between latitudes of $45 \mathrm{~N}$ and $45 \mathrm{~S}$. However, whether this a priori is valid for all latitudes is questionable. Therefore, we chose to extrapolate isothermally the temperature profiles for pressures lower than 0.2 mbar. Between 

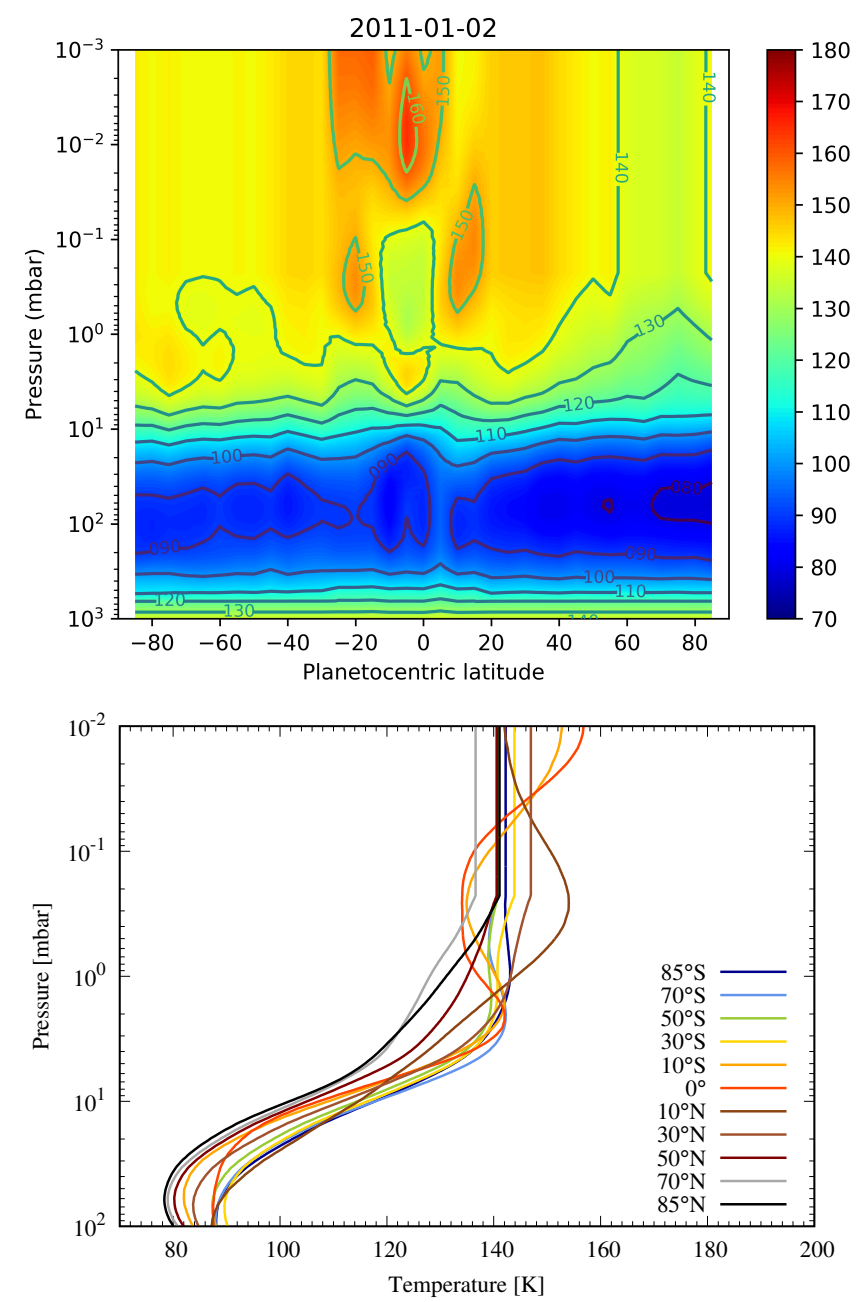

Fig. 4. Top: nominal thermal field used in this paper. Zonally averaged temperatures are given as a function of pressure and planetocentric latitude. The data combine retrievals from nadir and limb Cassini/CIRS data (see text for references). Bottom: temperature vertical profiles extracted from the field for a set of latitudes.

$20 \mathrm{~S}$ and 20N, we used the thermal profiles of February 2010 derived by Guerlet et al. (2011) from Cassini/CIRS limb viewing geometry observations to benefit from the higher vertical resolution of such observations. With such data, the equatorial quasi-periodic oscillation (Fouchet et al. 2008; Orton et al. 2008; Guerlet et al. 2011) is better resolved than in the nadir data, and the vertical sensitivity in the temperature retrieval is extended to $10^{-2}$ mbar. Figure 4 shows the thermal field. We note that the temperatures in the submillibar range are in agreement with temperatures derived from Voyager 2 occultation experiments at several latitudes by Lindal et al. (1985) for a similar $L_{\mathrm{S}}$.

\section{2. $\mathrm{H}_{2} \mathrm{O}$ distribution empirical models}

In this paper, we test two types of $\mathrm{H}_{2} \mathrm{O}$ spatial distributions that are representative of two different sources: IDPs and the Enceladus plumes. The IDP source is modeled with a meridionally uniform distribution of $\mathrm{H}_{2} \mathrm{O}$. The mole fraction of $\mathrm{H}_{2} \mathrm{O}$ is uniform above the local condensation level, which is computed following Fray \& Schmitt (2009) at each latitude. The Enceladus source is modeled with a two-parameter, Gaussian-shaped latitudinal distribution of $\mathrm{H}_{2} \mathrm{O}$, centered on the equator, which has a peak $\mathrm{H}_{2} \mathrm{O}$ mole fraction at the equator $y_{\text {eq }}$, and a half width at half maximum (HWHM) $\sigma$, such that

$y_{\mathrm{H}_{2} \mathrm{O}}(\phi)=y_{\text {eq }} \times \exp \left(-\frac{\phi^{2}}{2 \sigma^{2}}\right)$,

where $y_{\text {eq }}$ is the $\mathrm{H}_{2} \mathrm{O}$ mole fraction above the condensation level at the equator and $\phi$ is the planetocentric latitude. Latitudinally dependent condensation is computed from our thermal field. The recently observed ring source (Waite et al. 2018; Hsu et al. 2018; Mitchell et al. 2018) shares common properties with the Enceladus source: it is centered on the equator and has, to the first order, a Gaussian shape.

The ring atmosphere (mostly $\mathrm{O}_{2}$, not $\mathrm{H}_{2} \mathrm{O}$ ), another potential source for Saturn's stratospheric $\mathrm{H}_{2} \mathrm{O}$, has lower densities than the Enceladus neutral torus (Johnson et al. 2006; Cassidy \& Johnson 2010). Neutral-neutral collisions are therefore inefficient, and even ion-neutral charge exchange collisions are slow. Unlike elsewhere in the magnetosphere, the corotating plasma flow speed is similar to the orbital speed. The ring atmosphere is therefore lost at higher latitudes like ring rain: the atmosphere is lost via photoionization followed by precipitation along field lines (Moore et al. 2015). We note that the neutral component of their $\mathrm{H}_{2} \mathrm{O}$ distribution is tied to Enceladus. This is why we do not consider the ring atmosphere as a significant enough source to explain our observations and do not include this source in our models.

\subsection{Radiative-transfer model}

We modeled the observations with a line-by-line non-scattering model that solves the radiative-transfer equation in $1 \mathrm{D}$ on several thousand lines of sight, thus highly oversampling our observational spatial resolution. We used the spectral line parameters from the JPL Molecular Spectroscopy Database (Pickett et al. 1998). The spectra of collision-induced absorption caused by $\mathrm{H}_{2}-\mathrm{H}_{2}, \mathrm{H}_{2}-\mathrm{He}$, and $\mathrm{H}_{2}-\mathrm{CH}_{4}$ pairs were included following Borysow et al. $(1985,1988)$ and Borysow \& Frommhold (1986). More recent updates to the $\mathrm{H}_{2}-\mathrm{H}_{2}$ absorption from ab initio quantum theory exist (Orton et al. 2007; Fletcher et al. 2018a), but they do not make any significant difference in this spectral region. We adopted mole fractions of 11.8 and $0.47 \%$ for $\mathrm{He}$ and $\mathrm{CH}_{4}$ respectively, according to Conrath \& Gautier (2000) and Fletcher et al. (2009a). We accounted for the absorption caused by the $\mathrm{H}_{2} \mathrm{O}$ line as well as surrounding $\mathrm{NH}_{3}$ and $\mathrm{PH}_{3}$ broad lines. We took the $\mathrm{NH}_{3}$ and $\mathrm{PH}_{3}$ distributions from Davis et al. (1996) and Fletcher et al. (2009b), respectively. We computed the relevant $\mathrm{H}_{2} / \mathrm{He}$ broadening parameters from Levy et al. (1993, 1994) for $\mathrm{PH}_{3}$, from Fletcher et al. (2007) for $\mathrm{NH}_{3}$, and from Brown \& Plymate (1996) and Dick et al. (2009) for $\mathrm{H}_{2} \mathrm{O}$, for the $\mathrm{H}_{2} / \mathrm{He}$ mixture described above and the appropriate temperature range. The $1097 \mathrm{GHz}$ line has an opacity of $\sim 0.3$ over the HIFI beam in disk-centered conditions and the $66.44 \mu \mathrm{m}$ line has an opacity that ranges from 0.1 to 0.4 over the PACS beam for disk-centered and limb conditions for the best-fit model $\mathrm{H}_{2} \mathrm{O}$ profiles given in Sect. 4.4.

We note that, contrary to the $66.4 \mu \mathrm{m}$ line seen by PACS, the $1097 \mathrm{GHz}$ line seen by HIFI lies on the far wings of a $\mathrm{PH}_{3}$ line. A change in the $\mathrm{PH}_{3}$ abundance therefore influences the $l / c$ of the $\mathrm{H}_{2} \mathrm{O}$ line and subsequently on the derived $\mathrm{H}_{2} \mathrm{O}$ abundance. Increasing the $\mathrm{PH}_{3}$ abundance by a factor of 2 decreases the $\mathrm{H}_{2} \mathrm{O}$ abundance required to fit the line by $\sim 15 \%$.

This model significantly improves on the model already described in Cavalié et al. (2008b, 2013) regarding the handling of the geometry. The observation plane is sampled with an irregular grid so that the limb and rings, i.e., the regions with the 
highest variations in terms of continuum and line emission, are sufficiently sampled without hampering the computational efficiency. We accounted for the 3D ellipsoidal geometry of Saturn and rings at the time of the observations, using the parameters listed in Table 1. This means we computed the local latitudes, longitudes, and altitudes at each sampled location on each line of sight, and were thus able to use thermal and abundance fields that are fully 3D before applying the beam convolution.

In January 2011, the rings had an inclination of $12.4^{\circ}$, as seen from Herschel. We thus accounted for the contribution of the rings to the continuum emission. The A-B-C ring system brightness temperatures were computed in the wavelength range of our observations by interpolating the observational results obtained in the infrared with Cassini/CIRS (Flandes et al. 2010) and at $2 \mathrm{~cm}$ with the Very Large Array (van der Tak et al. 1999), accounting for the brightness temperature roll-off seen around $200 \mu \mathrm{m}$ by Spilker et al. (2003, 2005). We used the solar elevation as seen from the rings given in Table 1, and the optical depths of the different rings from Altobelli et al. (2014) and Guerlet et al. (2014). Our results are in general agreement with Dunn et al. (2005), when considering averages for the whole ring system. In practice, we geometrically identified the lines of sight that cross the rings and we accounted for the ring absorption and thermal emission, once all the lines of sight on the planet and limb are computed. Beyond the planetary disk, we found the positions of the rings and added their thermal emission. We then created a regular grid of lines of sight from which we computed the beam-averaged spectra in such a way that the beam is highly oversampled by the grid. We produced maps of line integrated emission (for PACS) and disk-averaged spectral line (for HIFI) that can directly be compared with the observation. We present the simulations as well as the residuals resulting from the subtraction of the model from the observations ${ }^{2}$.

\section{Results}

\subsection{Meridionally uniform distribution}

An initial approach was to try to fit the PACS map with a uniform distribution of $\mathrm{H}_{2} \mathrm{O}$ that would be representative of an IDP source. As shown in Fig. 5, the $\mathrm{H}_{2} \mathrm{O}$ line-area map is a direct translation of the thermal field and we find a strong gradient between the north and south poles. The minimum $\chi^{2} / N$ is obtained for an $\mathrm{H}_{2} \mathrm{O}$ mole fraction of $6 \times 10^{-10}$ above the condensation level (roughly a few millibar, varying with latitude), but the $\chi^{2} / N$ is as high as 18.9 (i.e., more than $4 \sigma$ away from the data). The observed decrease of the line area around the southern limb is not reproduced (see Fig. 5), as a results of the warmer stratospheric temperatures in the south compared to the north (see Fig. 4). On the contrary, the strongest emission comes from the south when we use a meridionally uniform distribution of $\mathrm{H}_{2} \mathrm{O}$. In addition, there is not enough emission at low latitudes. Such a solution is thus not satisfactory and can be discarded at this stage.

We note that an acceptable fit to the disk-averaged HIFI data can be obtained for a uniform mole fraction of $\sim 3.5 \times 10^{-9}$, which is consistent with Fletcher et al. (2012) but incompatible with the PACS data. When we simulate the $\mathrm{H}_{2} \mathrm{O}$ line at $556.936 \mathrm{GHz}$ with this distribution, we obtain a $l / c$ that is consistent with the previous observation of Bergin et al. (2000). This shows that the disk-averaged $\mathrm{H}_{2} \mathrm{O}$ influx responsible for

\footnotetext{
2 In the case of the HIFI spectrum, which was not absolutely calibrated, we have rescaled the spectrum to match our model continuum. The analysis is then done in terms of $l / c$.
}
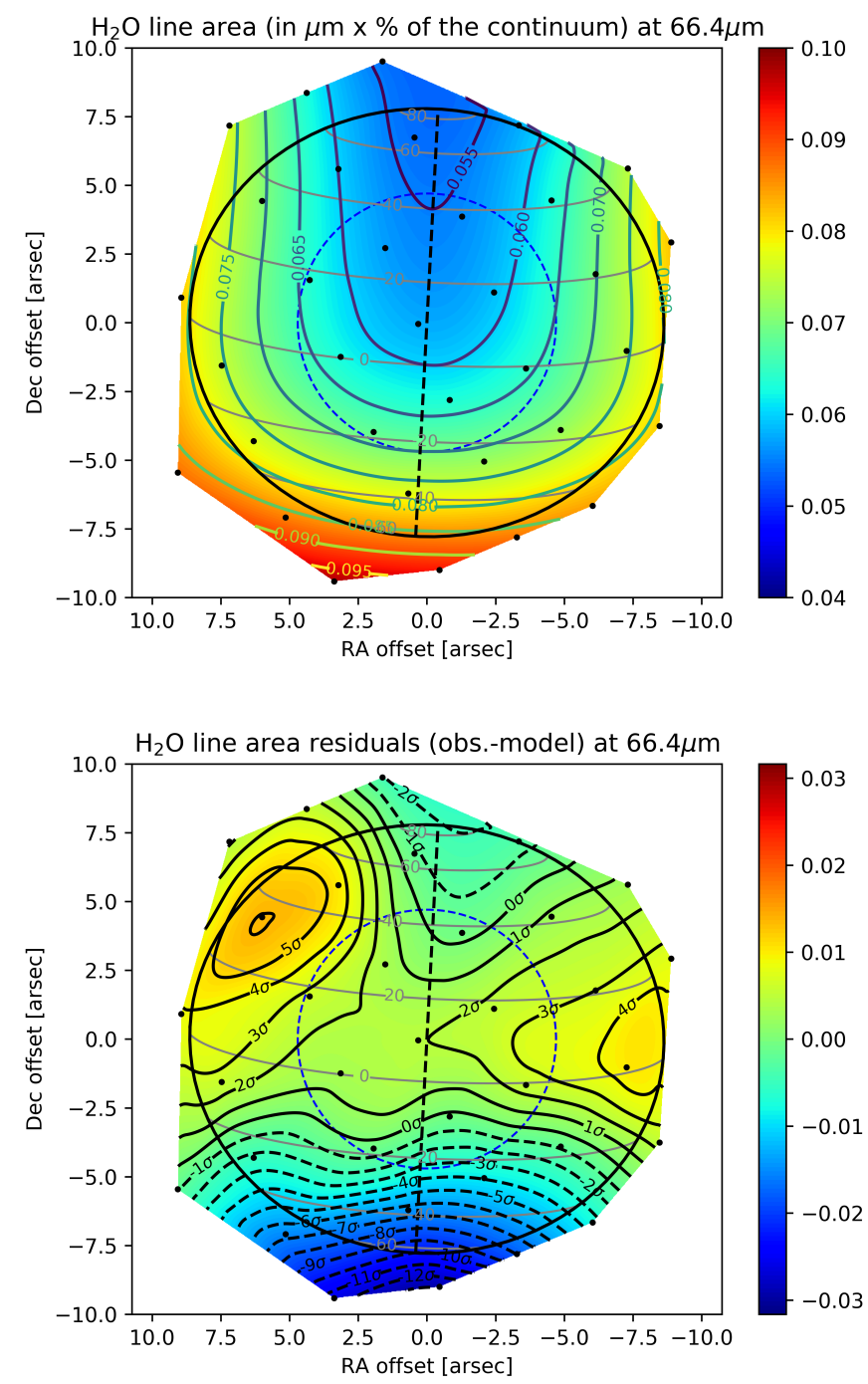

Fig. 5. Top: line-area map at $66.44 \mu \mathrm{m}$ modeled with a meridionally uniform distribution of $\mathrm{H}_{2} \mathrm{O}$. The line area is expressed in microns $\times$ percent of the continuum and can thus be directly compared to the observed map of Fig. 2. Bottom: map of the residuals between observations and model (in the same unit as the line-area map). Positive values indicate an excess of emission in the data compared to the model, while negative values indicate a lack of emission compared to the model. The contours are labeled with respect to the $1 \sigma$ noise level measured in Fig. 2. Above the condensation level, the $\mathrm{H}_{2} \mathrm{O}$ mole fraction is set to $6 \times 10^{-10}$. Neither the low latitudes nor the southern latitudes are within $3 \sigma$.

our observations of $\sim 6 \times 10^{5} \mathrm{~cm}^{-2} \mathrm{~s}^{-1}$ (Moses \& Poppe 2017), which translates into a disk-integrated mass flux of $\sim 8 \mathrm{~kg} \mathrm{~s}^{-1}$, is surpassed by orders of magnitude by the extraordinarily high flux recently measured by INMS of $\sim 10^{4} \mathrm{~kg} \mathrm{~s}^{-1}$ coming from the inner ring system. This proves that the ring source observed by Cassini in 2017 cannot be the cause of the $\mathrm{H}_{2} \mathrm{O}$ observed by Herschel in 2010-2011. The ring source must therefore be more recent than our observations, or at least this source had not been active for long enough (i.e., $~ 10-15 \mathrm{yr}$ ) to diffuse down to observable levels ( 0.1-1 mbar). According to Waite et al. (2018), such a large flux is probably linked to the appearance of clumps of material in the D68 ringlet in 2015 (Hedman \& Showalter 2016), and is unsustainable for more than $1 \mathrm{Myr}$ without depleting the rings. In conclusion, we will thus not consider the ring source in our subsequent modeling of the Herschel observations. 


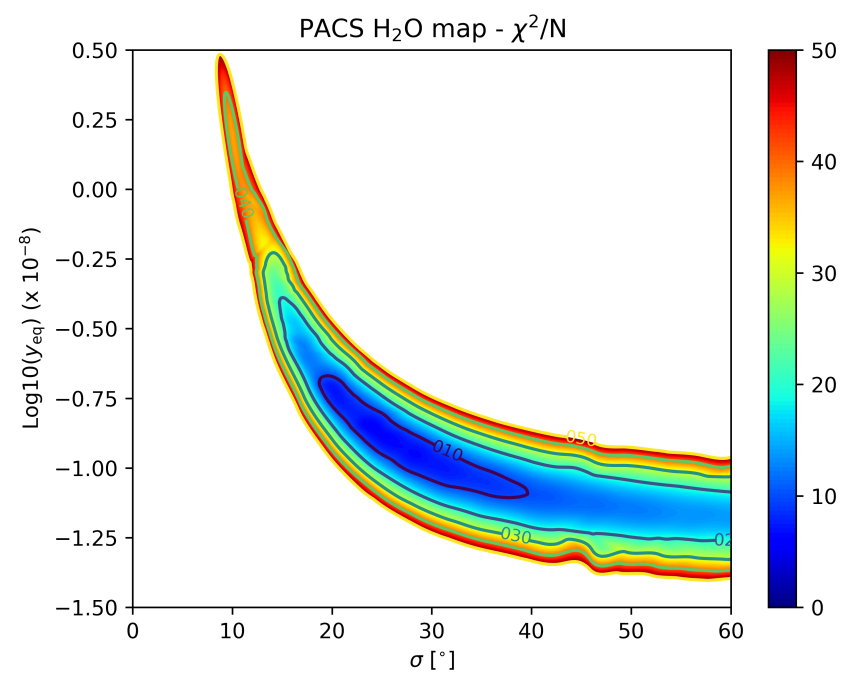

Fig. 6. $\chi^{2} / N$ as a function of the $\mathrm{H}_{2} \mathrm{O}$ meridional Gaussian distribution parameters $y_{\mathrm{eq}}$ and $\sigma$ (Gaussian HWHM in degrees). Acceptable solutions are found for $\sigma$ ranging from $\sim 20^{\circ}$ to $\sim 40^{\circ}$, with a minimum for $\sigma=25^{\circ}$ and $y_{\text {eq }}=1.4 \mathrm{ppb}$. Corresponding line area and residual maps are shown in Fig. 7.

\subsection{Meridionally variable distributions}

Despite the low spatial resolution of the PACS observations, the contrast observed between the eastern and western limbs and the northern and southern limbs (Fig. 2 bottom), combined with knowledge of the altitude and latitude thermal field (Fig. 4), provides us with a good tool for deriving the meridional distribution of $\mathrm{H}_{2} \mathrm{O}$ in Saturn's stratosphere.

In what follows, we test several cases of $\mathrm{H}_{2} \mathrm{O}$ meridional distributions as parametrized in Eq. (1). We first explore the $y_{\mathrm{eq}}-\sigma$ parameter space. Figure 6 shows the $\chi^{2} / N$ obtained as a function of these two parameters. There is a series of marginally acceptable solutions $\left(\chi^{2} / N<9\right)$. The best fit is obtained for $\sigma=25^{\circ}$ and $y_{\mathrm{eq}}=1.4 \mathrm{ppb}$, where $\chi^{2} / N=6.3$, and the corresponding line area and residual maps are shown in Fig. 7. We cannot obtain better overall fits with this model because of a remaining excess of emission in the northwestern limb (and to a lower extent in the northeastern limb). Minimizing the residuals implies compensating partially for this excess, which results in too much emission in the southern hemisphere. Thus, we first try to improve the fit by reassessing our temperature field in the next section, since the temperature field at the time of the observations was influenced by the onset of Saturn's Great Storm of 2010-2011.

As in the uniform distribution case, the HIFI data requires a $\sim 5$ times higher $y_{\text {eq }}$ than the PACS data for $\sigma=25^{\circ}$ to minimize $\chi^{2} / N$. The fit, shown in Fig. 8 left, is also only marginally acceptable. The line is too broad and not strong enough. This is an indication that models as simple as constant profiles above the condensation level are not optimal. The HIFI line-center contribution function peaks indeed at slightly lower pressures than the PACS line contribution function, which peaks near the condensation level (see Fig. 8). Introducing a positive gradient in the $\mathrm{H}_{2} \mathrm{O}$ vertical profile above the condensation layer should thus (i) reduce the width of the HIFI line by removing some $\mathrm{H}_{2} \mathrm{O}$ in the levels just above the condensation layer, (ii) increase the HIFI line amplitude by adding some $\mathrm{H}_{2} \mathrm{O}$ at higher levels to which the HIFI line center is still sensitive, and (iii) help to reconcile the HIFI and PACS data as they probe different altitudes.

In an attempt to improve the fit to the PACS data and to improve the compatibility with the HIFI data, we apply a
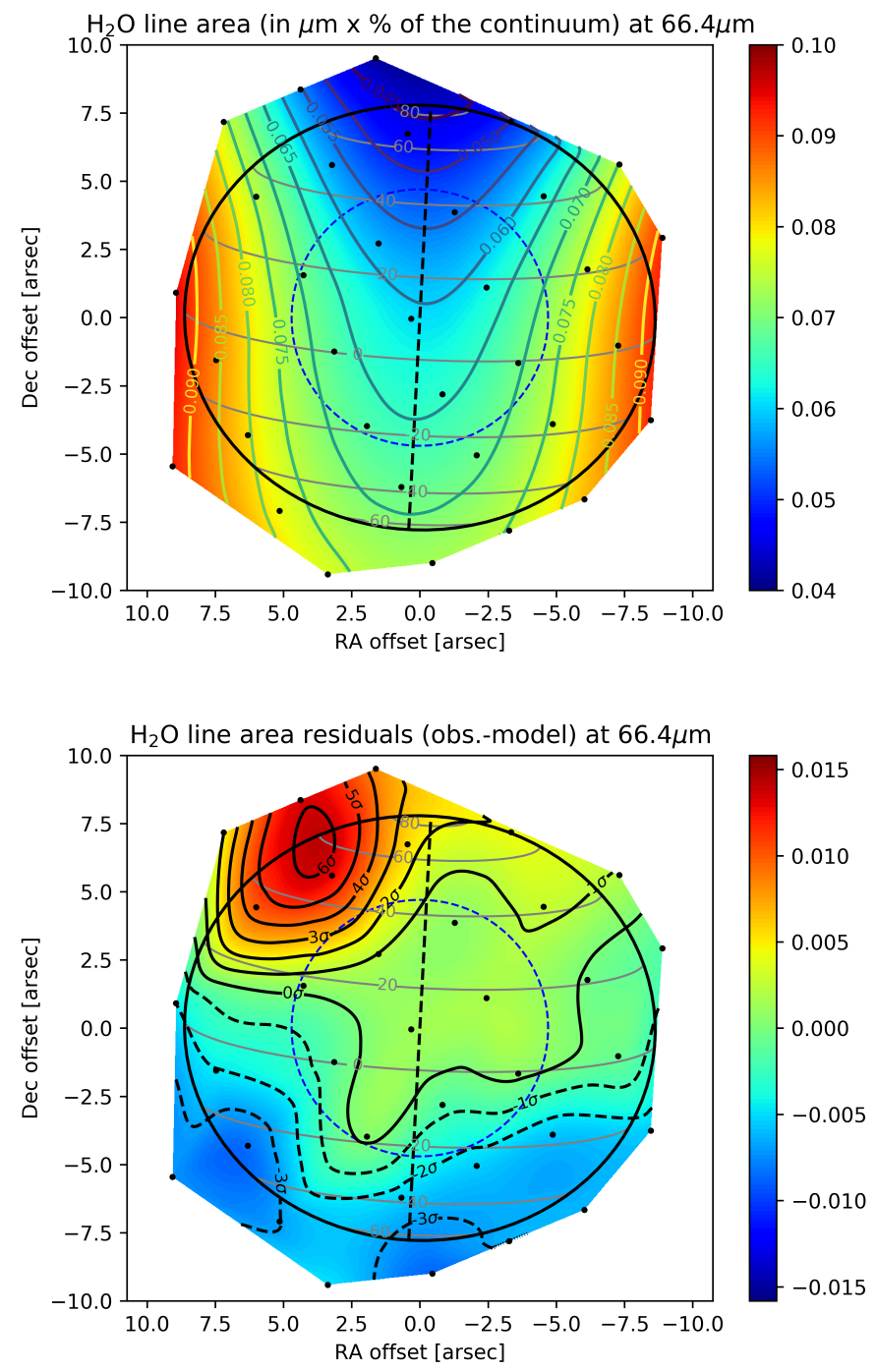

Fig. 7. Same as Fig. 5 for a Gaussian distribution of $\mathrm{H}_{2} \mathrm{O}$ around the equator with $y_{\mathrm{eq}}=1.4 \mathrm{ppb}$ and $\sigma=25^{\circ}$.

two-step approach, in which we first account for the temperature changes caused by Saturn's 2010-2011 Great Storm (Sect. 4.3), and then apply a parametrized gradient in the $\mathrm{H}_{2} \mathrm{O}$ vertical distribution above its condensation level (Sect. 4.4).

\subsection{Accounting for Saturn's Great Storm of 2010-2011}

The Herschel observations were conducted a few weeks after the formation of a huge storm system around $40^{\circ}$ in the northern hemisphere of the planet (Sánchez-Lavega et al. 2011; Fischer et al. 2011; Fletcher et al. 2011). Between December 2010 and April 2011, this storm system formed a cool region surrounded by two confined warm regions in Saturn's stratosphere. These are referred to as "beacons" (B1 and B2) given their appearance in the infrared combined with Saturn's fast rotation.

According to Cassini/CIRS observations made on the same day as the PACS data were recorded (and two days after the HIFI observations), the B1 and B2 beacons were located in the following longitude ranges: $300 \mathrm{~W}-340 \mathrm{~W}$ for B1 and $220 \mathrm{~W}-250 \mathrm{~W}$ for B2. The two beacons were thus both in the PACS field of view. Each region was located approximately halfway between the central meridian $\left(289^{\circ}\right)$ and the planetary limb (west for B1 and east for B2). Only B2 was in the HIFI field of view, located around the central meridian $\left(227^{\circ}\right)$. At that time, CIRS only observed the 

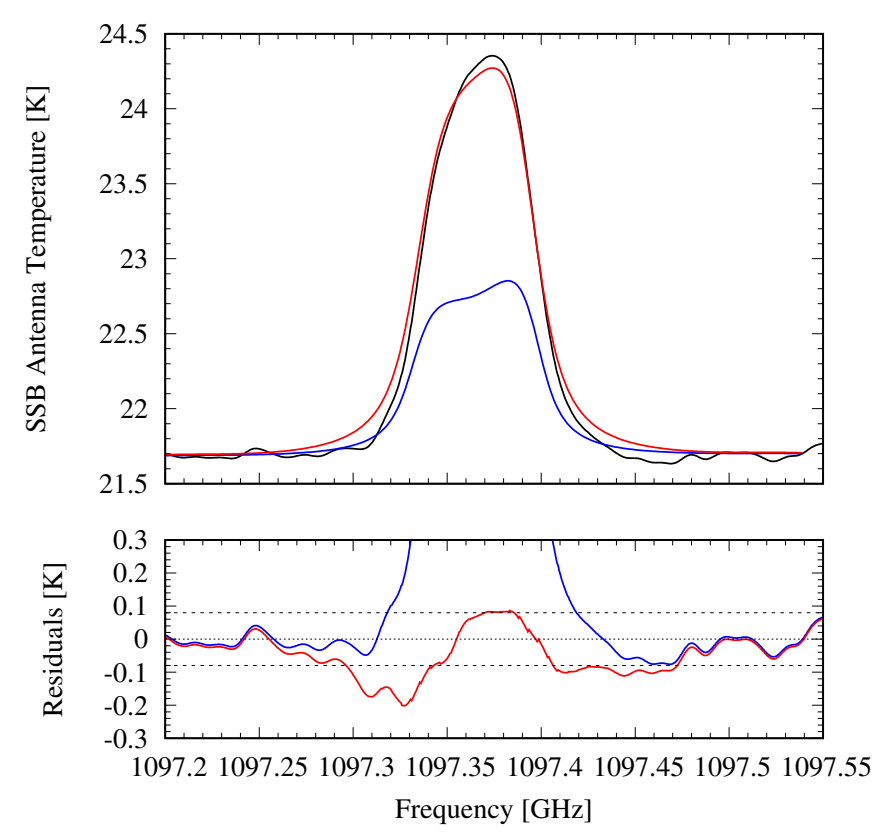

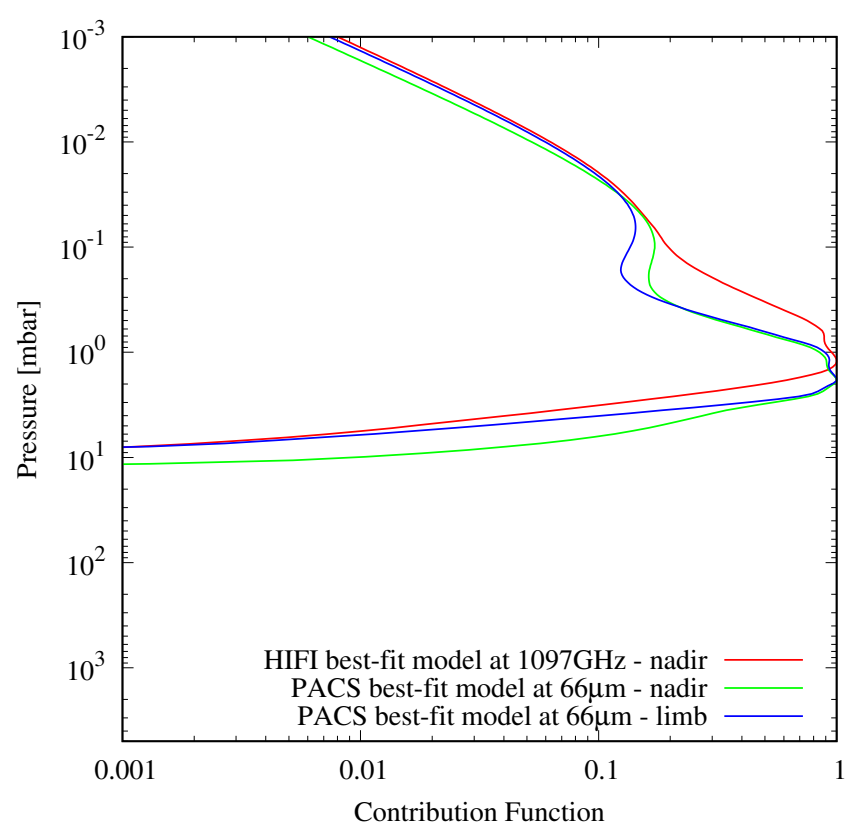

Fig. 8. Left: best fit to the HIFI data for a Gaussian distribution of $\mathrm{H}_{2} \mathrm{O}$ around the equator with $y_{\mathrm{eq}}=7.2 \mathrm{ppb}$ and $\sigma=25^{\circ}$. The data are shown in black and the model in red; the residuals (observation - model) are plotted at the bottom with the $1 \sigma$ level of noise in black dashed lines. The synthetic spectrum is too broad and there is a lack of emission at the center. The best-fit model to the PACS data from Fig. 7 is shown in blue for comparison. Right: normalized and beam-averaged contribution functions at the centers of the $66.44 \mu \mathrm{m}$ line in limb geometry (blue) and at the disk center (green), and of the $1097 \mathrm{GHz}$ line at the disk center (red) are shown. Both functions are computed at their respective observed spectral resolution and the contribution of the continuum emission was subtracted. We note that we rescaled the HIFI contribution function by multiplying it by a factor of 1.45 for an easier comparison with the PACS value.

northern edges of these beacons and an increase of $\sim 5-10 \mathrm{~K}$ had been measured there at the millibar level (Fletcher et al. 2012). Given the limited spatial coverage of the Cassini data and the absence of ground-based thermal data at that date, we assumed a latitudinal extent of $\mathrm{B} 1$ and $\mathrm{B} 2$ from $30^{\circ} \mathrm{N}$ to $50^{\circ} \mathrm{N}$ (planetocentric), and tried several temperature increase combinations within B1 and B2 compared to adjacent longitudes. As the emission excess seen in the data is more obvious in the northwest than in the northeast, we assume a stronger temperature increase in $\mathrm{B} 1$ compared to $\mathrm{B} 2$. We tested the following combinations: $+6 \mathrm{~K} /+3 \mathrm{~K},+10 \mathrm{~K} /+5 \mathrm{~K},+15 \mathrm{~K} /+10 \mathrm{~K}$, and $+20 \mathrm{~K} /+15 \mathrm{~K}$ for $\mathrm{B} 1 / \mathrm{B} 2$, respectively. We applied these temperature increases uniformly above the 10 mbar level at all latitudes/longitudes in $\mathrm{B} 1 / \mathrm{B} 2$, following the vertical trends in temperature found in these early development stages of B1 and B2 (see Fig. 8a in Fletcher et al. 2012). The choice of $10 \mathrm{mbar}$ as a cutoff pressure for the temperature increase was guided by the following argument: this level is below the $\mathrm{H}_{2} \mathrm{O}$ condensation level and thus we do not probe this level, while it is still well above the level where the continuum is generated. It is therefore a pragmatic way to ensure that the temperature changes in the beacon impacts the $\mathrm{H}_{2} \mathrm{O}$ lines. For $\mathrm{B} 1$ and $\mathrm{B} 2$, we also account for the longitudinal smearing of $15^{\circ}$ caused by the PACS integration time in the modeling of the thermal field. Our thermal field thus becomes a 3D field in our subsequent modeling (see Fig. 9), and we also recompute the condensation level of $\mathrm{H}_{2} \mathrm{O}$ in the beacons according to this new thermal field (see Fig. 10).

We obtain the best-fit results for the Gaussian meridional distribution of $\mathrm{H}_{2} \mathrm{O}$ with uniform temperature increases in $\mathrm{B} 1$ and $\mathrm{B} 2$ of 10 and $5 \mathrm{~K}$, respectively. The resulting 3D temperature field is shown in several latitudinal cross sections in Fig. 9. With this field, we significantly improve the fit to the PACS data, find a range of very good-fit $\left(\sigma, y_{\text {eq }}\right)$ combinations, and find a best fit $\left(\chi^{2} / N=1.1\right)$ for $\sigma=25^{\circ}$ and $y_{\text {eq }}=1.1 \mathrm{ppb}$. The line area and residual maps are shown in Fig. 11 and the column density as a function of latitude is shown in Fig. 12. We stress that we did not change the $\mathrm{H}_{2} \mathrm{O}$ abundance within the beacons in this work; we only changed the pressure of the condensation level. The overall fit is very good, even if, compared to our simulation, the results seem to indicate some enhancement in $\mathrm{H}_{2} \mathrm{O}$ abundance in the northwest limb from the excess of emission in the observations. Increasing the temperature further in B2 would improve the fit in this region, but degrade the fit at the north pole because of the large beam of PACS. In addition, the temperature profile within the beacons was more complex than our idealized isothermal model. Indeed, it had a peak at $0.5 \mathrm{mbar}$ and a drop at lower pressures (Fletcher et al. 2012). This should result in the need for more $\mathrm{H}_{2} \mathrm{O}$ in the millibar region to compensate for the fainter emission above the temperature peak. We leave this for future analysis of the beacon emissions.

With this model, we can even constrain a background and meridionally uniform flux (e.g., IDP) represented by a variable $y_{\text {min }}$ that can be added to Eq. (1). We find that it cannot exceed $0.06 \mathrm{ppb}$ at the $2 \sigma$ limit (Fig. 13), which is an order of magnitude lower than the disk-averaged contribution of the Gaussian distribution. This confirms the small contribution from IDP, as predicted by Moses \& Poppe (2017). New photochemical simulations are now required to determine the corresponding IDP $\mathrm{H}_{2} \mathrm{O}$ flux upper limit.

Accounting for the temperature increase in B2, which is in the HIFI field of view does not improve the situation regarding the PACS/HIFI incompatibility, i.e., $\sim 5$ times more $\mathrm{H}_{2} \mathrm{O}$ than in the PACS best-fit model is needed to fit the HIFI line. The best-fit model remains unchanged in terms of $y_{\mathrm{eq}}$ for a given $\sigma$, compared to Sect. 4.2, and the fit to the HIFI data has the same flaws in that the wings are too broad and line center is too weak. 

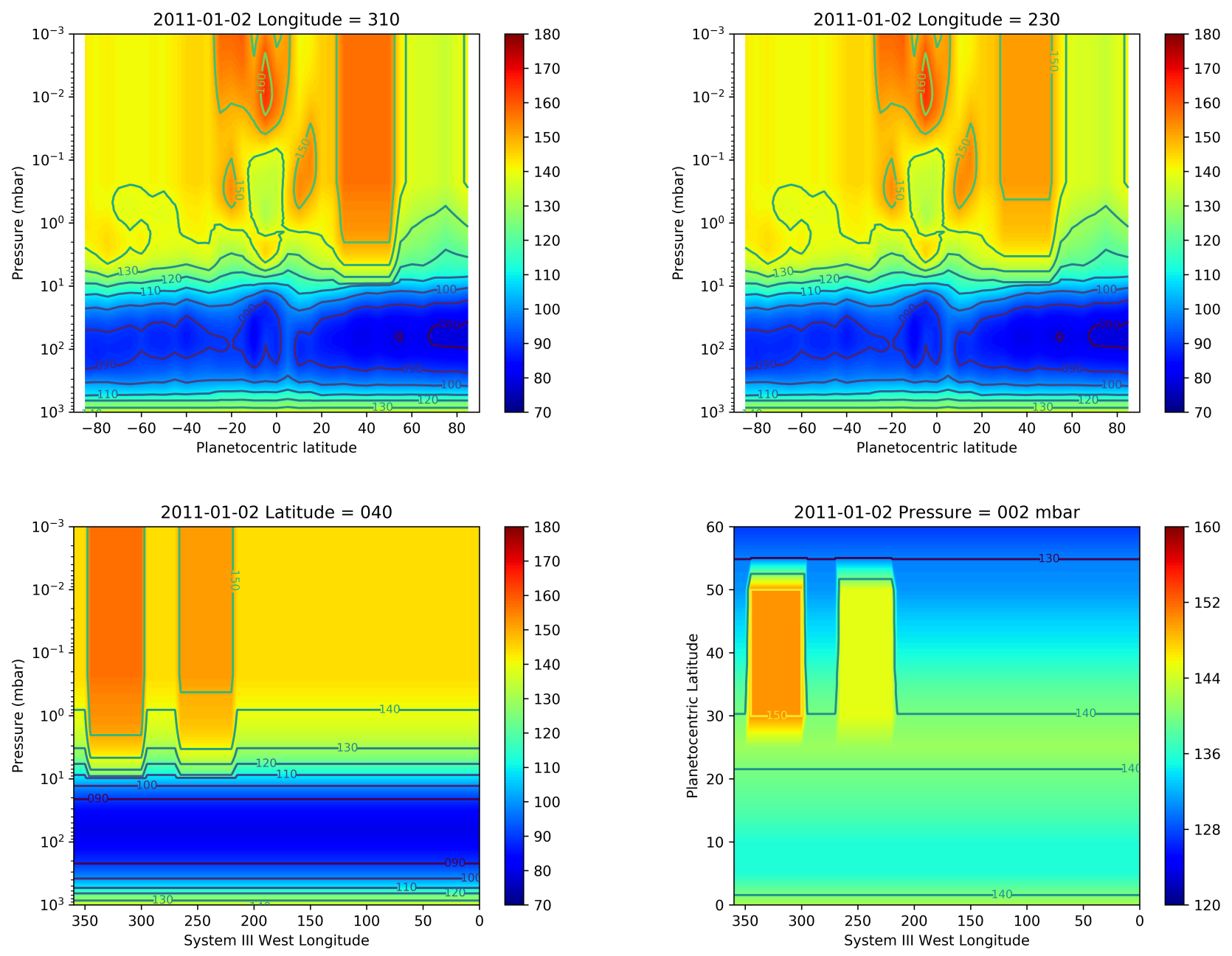

Fig. 9. Three-dimensional thermal field used in simulations accounting for $10 \mathrm{~K}$ temperature increases in $\mathrm{B} 1$ and $5 \mathrm{~K}$ for $\mathrm{B} 2$. B1 is located between $300 \mathrm{~W}-355 \mathrm{~W}$; B2 is located between 220W-265W when accounting for longitudinal smearing during the PACS integration; and both are located between $30 \mathrm{~N}$ and $50 \mathrm{~N}$ (Fletcher et al. 2012). Top panels: meridional cross sections isolating B1 (left) and B2 (right). Bottom left panel: zonal cross section at $40 \mathrm{~N}$. Bottom right: pressure cross section at 2 mbar, in which both beacons can be identified.

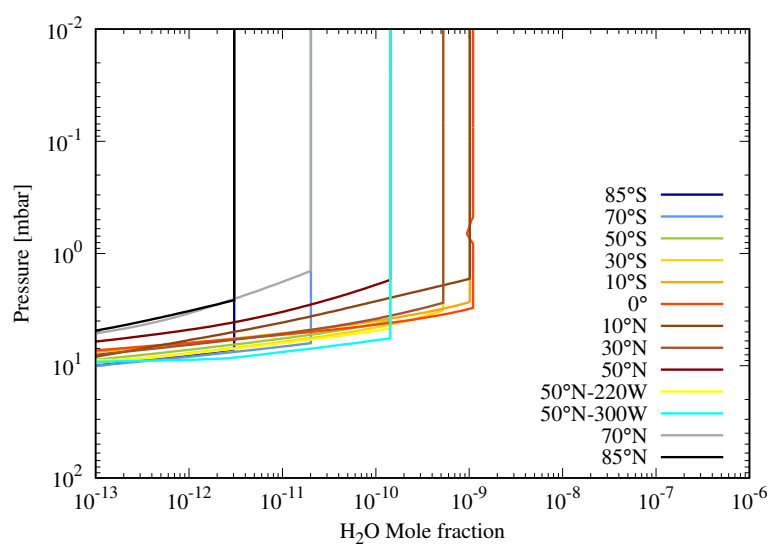

Fig. 10. $\mathrm{H}_{2} \mathrm{O}$ vertical profiles of the PACS best-fit model (see Fig. 11), in which the latitudinal distribution is a Gaussian centered around the equator with $\sigma=25^{\circ}$, and $y_{\mathrm{eq}}=1.1 \mathrm{ppb}$. The effect of the temperature increases in the B1 and B2 beacons is accounted for when computing the condensation level, as shown by the yellow and cyan lines (corresponding to $\mathrm{B} 1$ and $\mathrm{B} 2$, respectively). The local equatorial minimum at 1 mbar results from condensation at a temperature minimum caused by the Saturn quasi-periodic oscillation (see Fig. 4). The corresponding line area and residual maps are shown in Fig. 11.
Now that we have a good fit to the PACS data, we have to briefly turn back to the meridionally uniform distribution model to check whether the improvement in the temperature field induces any improvement in the fit to the data for such a thermal model. Figure 14 shows the line area and residual map obtained for an $\mathrm{H}_{2} \mathrm{O}$ mole fraction of $4 \times 10^{-10}$ above the condensation level. With a $\chi^{2} / N=16.2$, such a model remains invalid. After leveling out the thermal effects from the line emission map, the uniform distribution still results in high latitudes being too bright and low latitudes being too faint compared to the observations.

Accounting for the effect on the thermal field of Saturn's Great Storm of 2010-2011 has enabled us to significantly improve the quality of the fit to the PACS data with the Gaussian meridional $\mathrm{H}_{2} \mathrm{O}$ distribution and confirmed the invalidity of the meridionally uniform distribution.

\subsection{Introducing a gradient in the $\mathrm{H}_{2} \mathrm{O}$ vertical profile above its condensation layer}

In this section, we seek to reconcile the PACS and HIFI observations while building on the improvements in the PACS interpretation, which resulted from including Saturn's Great Storm effect in our modeling, by introducing a gradient in the $\mathrm{H}_{2} \mathrm{O}$ vertical distribution above the condensation level. 

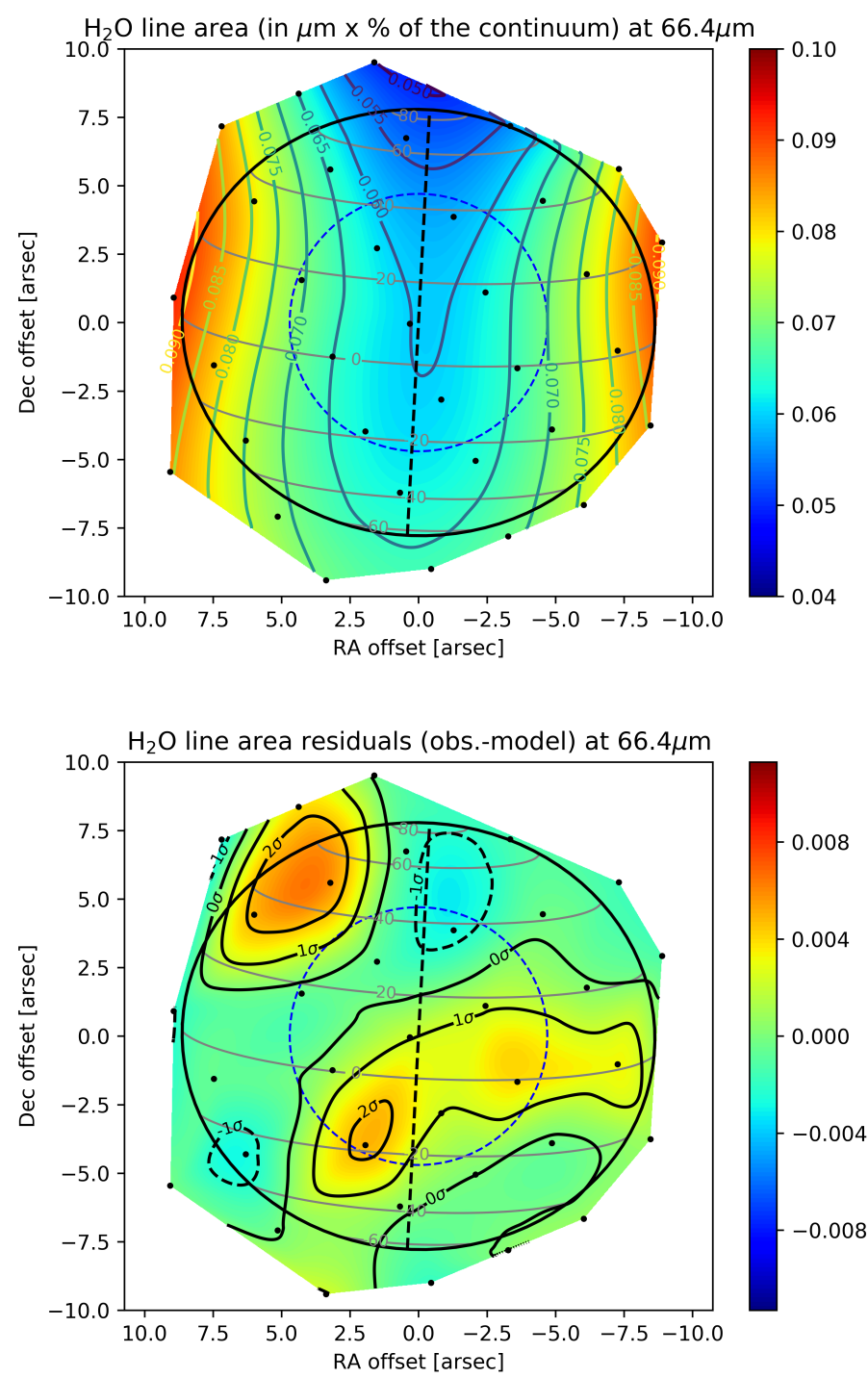

Fig. 11. Same as Fig. 5 for a Gaussian distribution of $\mathrm{H}_{2} \mathrm{O}$ around the equator with $y_{\mathrm{eq}}=1.1 \mathrm{ppb}$ and $\sigma=25^{\circ}$, after accounting for the temperature increases in the B1 and B2 stratospheric beacons caused by Saturn's Great Storm of 2010-2011 at the time of the PACS observations.

As the HIFI line probes slightly higher than the PACS line, and as the HIFI line is too broad in the wings and too faint at line center, we introduce a parametrized vertical gradient in the $\mathrm{H}_{2} \mathrm{O}$ profile at all latitudes to make it less abundant above the condensation level and more abundant at higher altitude. This way, the empirical $\mathrm{H}_{2} \mathrm{O}$ vertical profiles are closer to physical profiles computed with photochemical models (e.g., Ollivier et al. 2000; Moses et al. 2000). As the PACS data require less $\mathrm{H}_{2} \mathrm{O}$ than the HIFI data, we see this gradient as a means to reconcile the two datasets. To achieve this, we introduce two additional parameters: a cutoff pressure $p_{\text {gradient }}$, above which the $\mathrm{H}_{2} \mathrm{O}$ abundance is held constant, and $n$ which is the $\log (y) / \log (p)$ slope of the profile between the cutoff pressure and the condensation level, as already used in previous works (Marten et al. 2005; Rezac et al. 2014). We test several combinations of $p_{\text {gradient }}$ and $n$ : values of $p_{\text {gradient }}$ range from 0.01 to 1 mbar, where $p_{\text {gradient }}$ is always smaller than the pressure of the condensation level, whatever the latitude; and values of $n$ range from 0.5 to 3 (an example is shown in Fig. 15).

We find that adding a positive gradient in the $\mathrm{H}_{2} \mathrm{O}$ vertical profile above the condensation layer reduces the incompatibility between the HIFI and PACS $y_{\text {eq }}$ values from a factor of $\sim 5$ down to a factor of $\sim 2.4$ for $n=2-3$ and $p_{\text {gradient }}<0.3$ mbar in the range of values we tested. An example for PACS is shown in Fig. 16 for $n=2, p_{\text {gradient }}=0.1 \mathrm{mbar}, y_{\mathrm{eq}}=9 \times 10^{-8}$ and $\sigma=25^{\circ}$. The corresponding vertical profiles as a function of latitude are shown in Fig. 15. The corresponding best fit of the HIFI line is obtained for the same set of parameters, except $y_{\mathrm{eq}}=2.2 \times 10^{-7}$ (see Fig. 17).

\subsection{Final remarks on the HIFI/PACS discrepancy}

As it stands, we find no $\mathrm{H}_{2} \mathrm{O}$ distribution that enables us to fully reconcile the HIFI and PACS data in terms of $\mathrm{H}_{2} \mathrm{O}$ abundance (through the value of $y_{\mathrm{eq}}$ ) even if introducing a positive gradient above the condensation level significantly improves the situation.

There are some systematic and random errors we have not considered that may explain at least in part the remaining discrepancy between the HIFI and PACS derived abundances. For instance, there is some scatter in the $\mathrm{PH}_{3}$ disk-averaged abundances derived from observations (e.g., Weisstein \& Serabyn 1996 vs. Fletcher et al. 2012), its meridional distribution was only measured once by Fletcher et al. (2009b) and any temporal evolution is therefore unconstrained. As noted in Sect. 3.3, the $\mathrm{PH}_{3}$ distribution influences the continuum of the $1097 \mathrm{GHz}$ line (but not of the $66.4 \mu \mathrm{m}$ line), and thus significantly influences the derivation of the $\mathrm{H}_{2} \mathrm{O}$ abundance from this line. The thermal field also bears some uncertainties $(\sim 3 \mathrm{~K}$ at the tropopause and $\sim 4 \mathrm{~K}$ at $1-5 \mathrm{mbar}$, as detailed in Fletcher et al. 2018b). Given that we implement a full 3D thermal field and that the opacities of the observed $\mathrm{H}_{2} \mathrm{O}$ lines are of the same order but not strictly identical, the uncertainties on the thermal field may be an additional cause of discrepancy between the HIFI and PACS lines.

Finally, we stress that the meridional distribution may be more complicated than that given by Eq. (1), and the vertical gradient may also vary with latitude. This study can thus be seen as a stepping stone for future computations of physical profiles with more complete altitude-latitude photochemical models.

\section{Discussion}

In situ measurements carried out by several Cassini instruments during the end-of-mission orbits and final plunge into the atmosphere (all in 2017) have provided strong evidence for material infalling from the rings onto Saturn's atmosphere whether in gaseous or solid form, or neutral or ionized (Hsu et al. 2018; Mitchell et al. 2018; Waite et al. 2018). While small infalling icy grains, constrained to $\pm 2^{\circ}$ around the equatorial plane rain onto Saturn with a modest mass flux of $5 \mathrm{~kg} \mathrm{~s}^{-1}$ (Mitchell et al. 2018), $\mathrm{H}_{2} \mathrm{O}$ gas seems to be feeding Saturn's upper atmosphere at a much higher rate. From INMS on board Cassini, Waite et al. (2018) have found that a global integrated mass flux of 4800 $45000 \mathrm{~kg} \mathrm{~s}^{-1}$ of gaseous material was raining onto the planet's atmosphere at the time of the measurements, with $24 \pm 5 \% \mathrm{H}_{2} \mathrm{O}$, within a latitudinal band of $8^{\circ}$ centered on the equator. Using data from the same instrument, Perry et al. (2018) have found an even larger influx of $2-20 \times 10^{4} \mathrm{~kg} \mathrm{~s}^{-1}$. This is $2-4$ orders of magnitude higher than the influx of $\mathrm{H}_{2} \mathrm{O}$ of $7-25 \mathrm{~kg} \mathrm{~s}^{-1}$ derived from previous disk-averaged observations and models (Feuchtgruber et al. 1997; Bergin et al. 2000; Moses et al. 2005; Hartogh et al. 2011). Therefore, this material infalling from the inner ring system, seen in 2017 by Cassini, cannot be the source of the $\mathrm{H}_{2} \mathrm{O}$ observed by Herschel in December 2010 and January 2011.

An earlier Cassini finding provided another credible candidate: Enceladus and its $\mathrm{H}_{2} \mathrm{O}$ plumes (Hansen et al. 2006; 


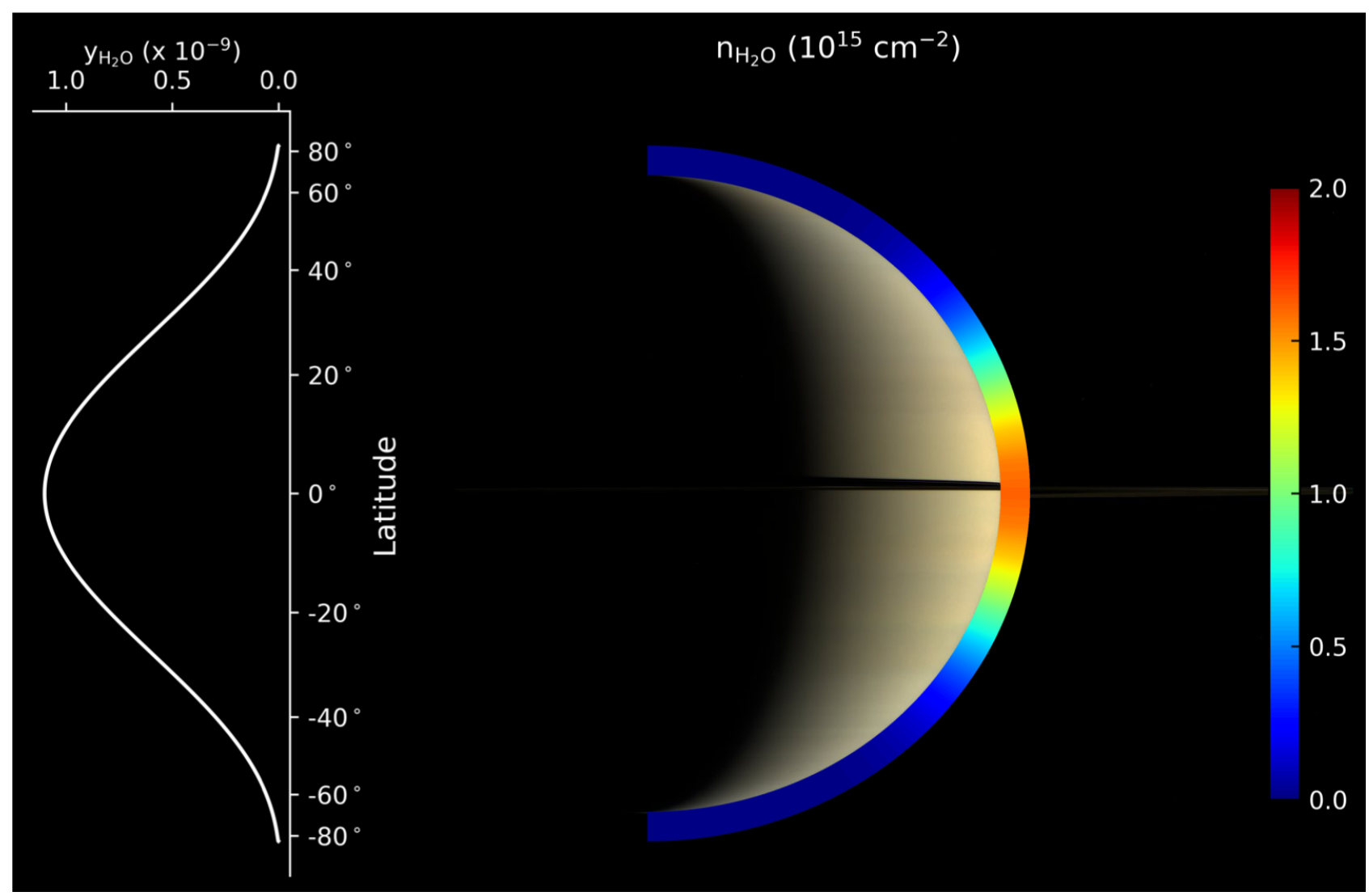

Fig. 12. Saturn's stratospheric $\mathrm{H}_{2} \mathrm{O}$ meridional distribution as derived from Herschel-PACS mapping observations on January 2, 2011. Left: mole fraction above the local condensation level as a function of latitude. Right: corresponding column density as a function of latitude. The distribution is a Gaussian centered on the equator with $y_{\mathrm{eq}}=1.1 \mathrm{ppb}$ and $\sigma=25^{\circ}$, corresponding to the PACS map best-fit model (see Fig. 11). Background image credits: NASA/JPL-Caltech/Space Science Institute.

Porco et al. 2006; Waite et al. 2006). Cassini's first observations of the plumes at the south pole of the moon venting out significant amounts of $\mathrm{H}_{2} \mathrm{O}$ triggered a modeling effort to evaluate the fate of the outgassed molecules (Jurac \& Richardson 2007; Cassidy \& Johnson 2010; Moore et al. 2010). For instance, Cassidy \& Johnson (2010) found that neutral/neutral scattering was the main cause of spreading of the molecules in Saturn's system and predicted that Enceladus plumes formed an $\mathrm{H}_{2} \mathrm{O}$ neutral torus at the orbital distance of the satellite. This torus was confirmed by the observations of Hartogh et al. (2011). According to Cassidy \& Johnson (2010), this torus is likely to be a significant source of $\mathrm{H}_{2} \mathrm{O}$ for Saturn's rings, the planet itself, and even Titan. The latter was confirmed by Moreno et al. (2012). At Saturn, the meridional distribution of infalling neutral $\mathrm{H}_{2} \mathrm{O}$ originating from Enceladus plumes is predicted by Cassidy \& Johnson (2010) to be Gaussian and centered on the planet equator with a $H W H M \sigma \sim 15^{\circ}$. The disk-averaged mass flux was estimated to $6 \times 10^{5} \mathrm{~cm}^{-2} \mathrm{~s}^{-1}$, i.e., $\sim 8 \mathrm{~kg} \mathrm{~s}^{-1}$, by Hartogh et al. (2011) by fitting the Enceladus source to the absorption caused by the Enceladus torus on the Saturn $\mathrm{H}_{2} \mathrm{O}$ line at $557 \mathrm{GHz}$. Comparing this $6 \times 10^{5} \mathrm{~cm}^{-2} \mathrm{~s}^{-1}$ flux to the required external flux invoked to explain Saturn's water $(1 \pm 0.5) \times 10^{6}$ (Moses et al. 2000), Hartogh et al. (2011) concluded that the Enceladus plumes were the likely source of Saturn's stratospheric $\mathrm{H}_{2} \mathrm{O}$.

In this paper, we have analyzed a Herschel-PACS map of $\mathrm{H}_{2} \mathrm{O}$ emission coming from Saturn's stratosphere. We presented clear evidence that $\mathrm{H}_{2} \mathrm{O}$ in Saturn's stratosphere is not meridionally uniform and that a distribution in which its abundance peaks at the equator and decreases exponentially toward higher latitudes fits the data. This result enables us to identify Enceladus as the main source of Saturn's stratospheric $\mathrm{H}_{2} \mathrm{O}$, and to reject the IDP as a primary source. However, a faint meridionally uniform background flux cannot be ruled out by our observations. Our data indicate that this background is an order of magnitude lower than the disk-averaged contribution of the Enceladus source, which is in agreement with the prediction from Moses \& Poppe (2017).

The difference we find between the predicted width of the influx meridional distribution with that observed allows us to compute a rough estimate of meridional eddy mixing at lowto-mid latitudes. If we assume that $K_{y y} \sim L^{2} / t$, we then take $L \sim 10500 \mathrm{~km}$ as the distance between $15^{\circ}$ latitude and $25^{\circ}$ latitude. These latitudes correspond to the HWHMs of the input flux from Cassidy \& Johnson (2010) and our best model. For the diffusion timescale between those latitudes, we take $t$ equal to the downward diffusion timescale from the top of the atmosphere, where the material is delivered, to the condensation level (150y according to Moses \& Poppe 2017), where $\mathrm{H}_{2} \mathrm{O}$ was mapped by Herschel. We find that $K_{y y} \sim 2 \times 10^{8} \mathrm{~cm}^{-2} \mathrm{~s}^{-1}$, and this value is nominally lower by a factor of 10 than early modeling results from Friedson \& Moses (2011). A more accurate derivation of the input fluxes as a function of latitude at Saturn that are responsible for the observations will require additional simulation work with 2D photochemical models (Hue et al. 2015, 2016, 2018).

\section{Conclusion}

The findings of our paper can be summarized as follows:

- We mapped Saturn's stratospheric $\mathrm{H}_{2} \mathrm{O}$ emission with Herschel-PACS on January 2, 2011 and obtained a 

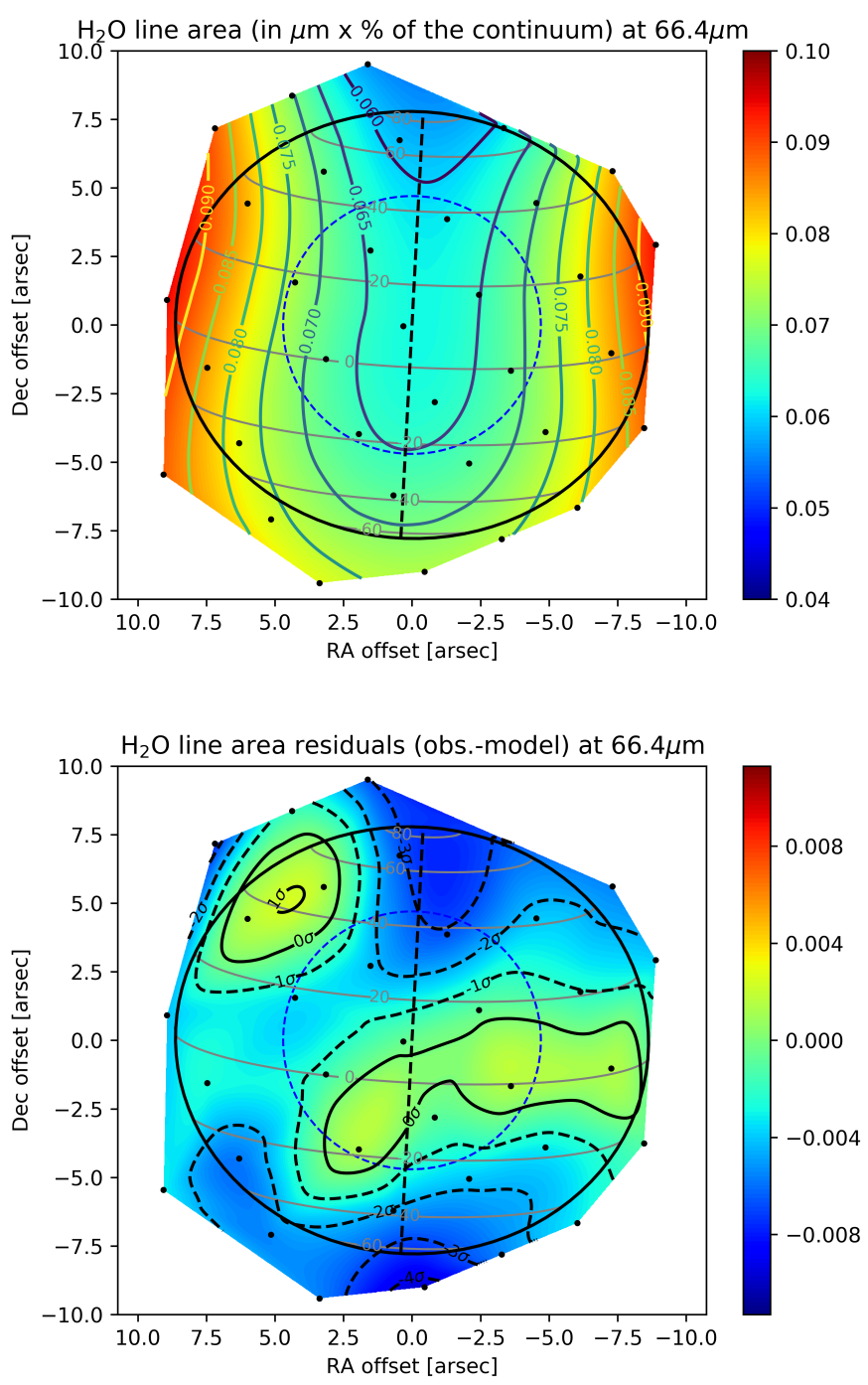

Fig. 13. Same as Fig. 11, in which a uniform component is added to the Gaussian distribution of $\mathrm{H}_{2} \mathrm{O}$. The model distribution parameters are $y_{\mathrm{eq}}=1.04 \mathrm{ppb}, \sigma=25^{\circ}$, and $y_{\min }=0.06 \mathrm{ppb}$. The overall fit is degraded compared to Fig. 11 because of the uniform component of the distribution.

disk-averaged spectrum of the $\mathrm{H}_{2} \mathrm{O}$ line at $1097 \mathrm{GHz}$ with Herschel-HIFI on December 31, 2010.

- We find that a meridionally uniform distribution of $\mathrm{H}_{2} \mathrm{O}$ above its condensation level does not fit the data, which implies that IDP are not the primary source of Saturn's stratospheric $\mathrm{H}_{2} \mathrm{O}$.

- We find that the data are reasonably well reproduced with a meridional distribution of $\mathrm{H}_{2} \mathrm{O}$ that is Gaussian-shaped and centered around the equator, when accounting for the $3 \mathrm{D}$ temperature field at the time of our observations, including the effect of Saturn's Great Storm of 2010-2011. The best fit is obtained for an equatorial mole fraction of $1.1 \mathrm{ppb}$ and a HWHM of $25^{\circ}$. This type of distribution points to the Enceladus plumes as the primary source of Saturn's observed stratospheric $\mathrm{H}_{2} \mathrm{O}$.

- We can place an upper limit on a meridionally uniform background source like IDP about 10 times fainter than the main equatorial source, in agreement with theoretical predictions by Moses \& Poppe (2017).

- We can improve the compatibility between the results obtained from the HIFI and PACS observations by adding
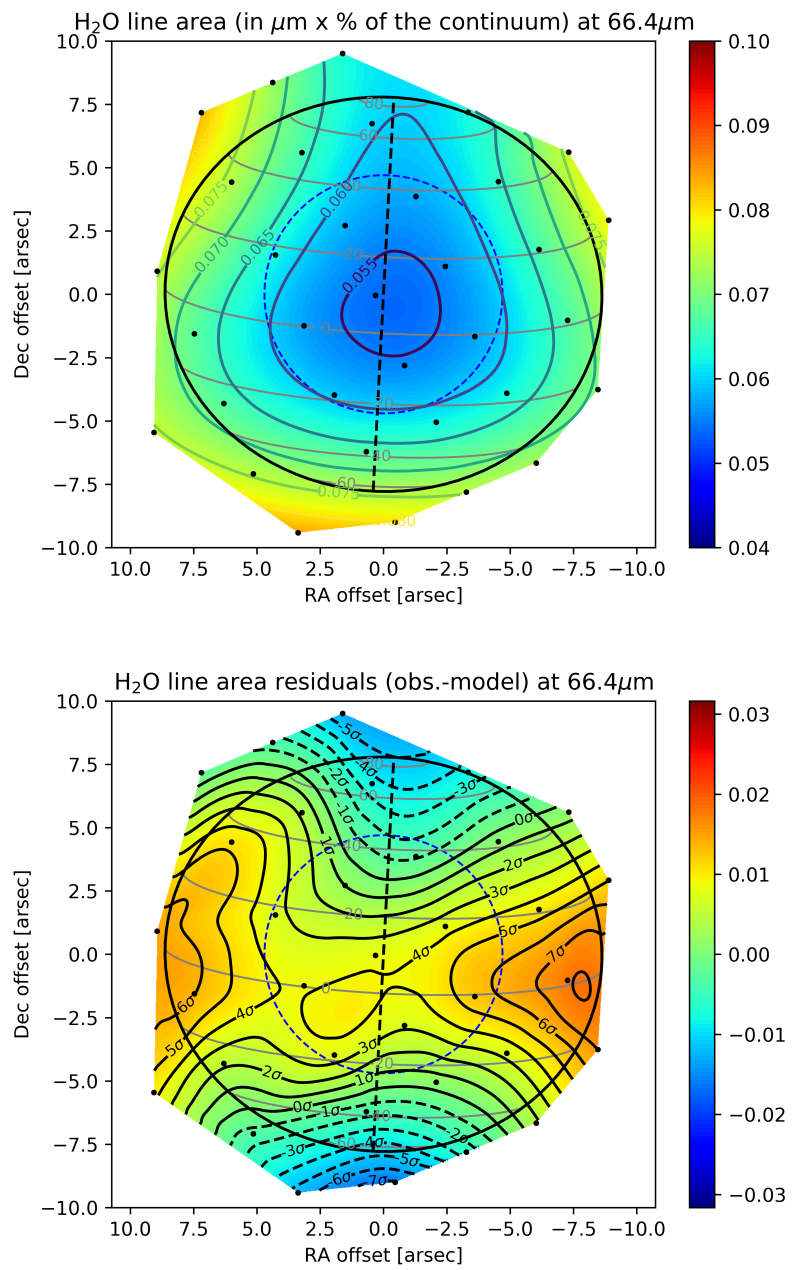

Fig. 14. Same as Fig. 5 for a meridionally uniform distribution of $\mathrm{H}_{2} \mathrm{O}$ with a mole fraction set to $4 \times 10^{-10}$, after accounting for the temperature increases in the B1 and B2 stratospheric beacons caused by Saturn's Great Storm of 2010-2011 at the time of the PACS observations. The overall fit is still poor $\left(\chi^{2} / N=16.2\right)$ such that accounting for the temperature increases caused by Saturn's Great Storm of 2010-2011 does not reconcile such an $\mathrm{H}_{2} \mathrm{O}$ distribution with the data.

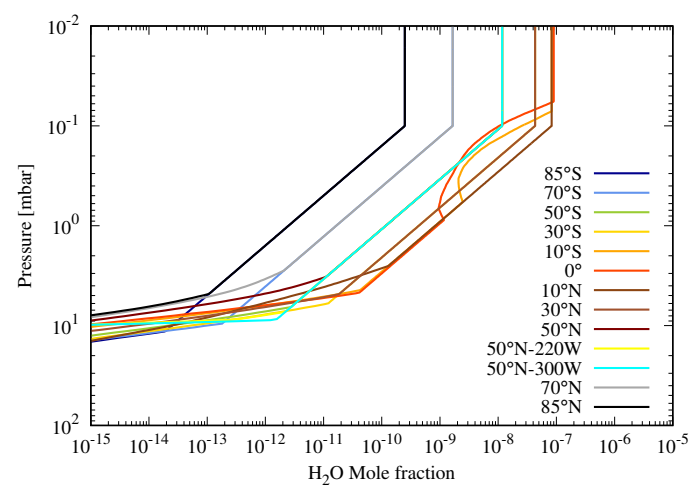

Fig. 15. $\mathrm{H}_{2} \mathrm{O}$ vertical profiles of the PACS fit model of Fig. 16, in which the latitudinal distribution is a Gaussian centered around the equator with $\sigma=25^{\circ}$, and $y_{\mathrm{eq}}=9 \times 10^{-8}$. The effect of the temperature increases in the B1 and B2 beacons is accounted for when computing the condensation level, and a positive gradient is added for pressures higher than $p_{\text {gradient }}=0.1 \mathrm{mbar}$ and down to the condensation level, with a slope $n=\log (y) / \log (p)=2$. The value of $y_{\text {eq }}$ applies to $p<p_{\text {gradient }}$. Condensation between 0.1 and 1 mbar occurs in the equatorial region as a result of a local temperature minimum caused by the quasi-periodic oscillation (see Fig. 4). The corresponding line area and residual maps are shown in Fig. 16. 

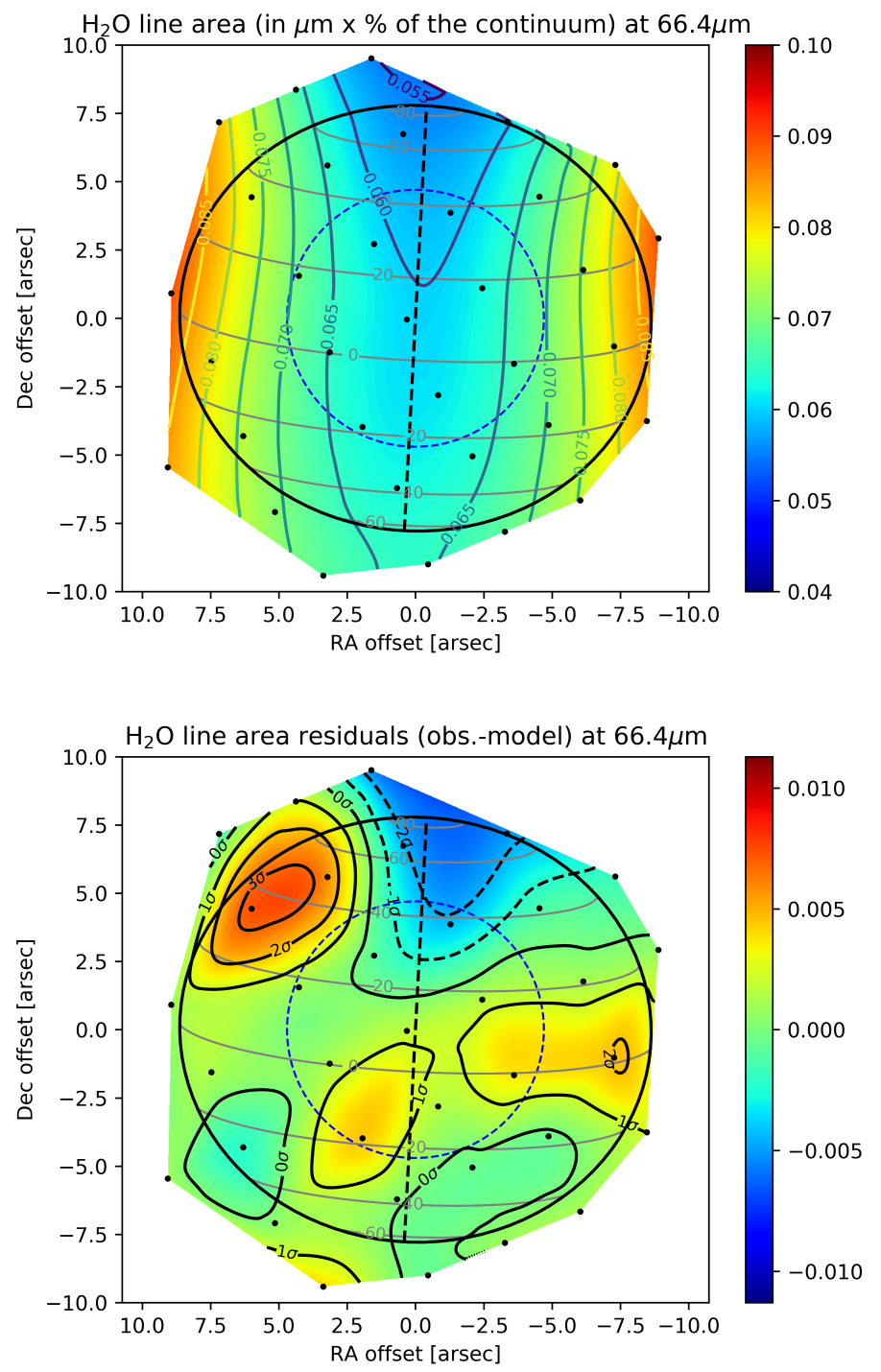

Fig. 16. Same as Fig. 5 for a Gaussian distribution of $\mathrm{H}_{2} \mathrm{O}$ around the equator with $y_{\mathrm{eq}}=9 \times 10^{-8}$ and $\sigma=25^{\circ}$, after accounting for the temperature increases in the B1 and B2 stratospheric beacons caused by Saturn's Great Storm of 2010-2011 at the time of the PACS observations, and a positive gradient in the $\mathrm{H}_{2} \mathrm{O}$ profile above the condensation level (with $n=2$ and $p_{\text {gradient }}=0.1$ mbar). More details about the vertical profiles can be found in Fig. 15. The overall fit remains good compared to Fig. 11, and facilitates reducing the inconsistency between the $y_{\mathrm{eq}}$ values derived from PACS and HIFI to a factor of $\sim 2$.

a positive gradient above the condensation level in the $\mathrm{H}_{2} \mathrm{O}$ vertical profiles, in an attempt to bring our profiles closer to physical profiles. However, we do not manage to fully reconcile the disk-resolved PACS data and the disk-averaged HIFI data with our empirical models, probably because of remaining systematic and random errors and because of the simplicity of our meridional distribution model. Full 2D photochemical modeling is now necessary to move to the next step.

It remains to be seen what meridional distribution of input fluxes are required to reproduce the Herschel observations, and 2D photochemical modeling is required at this stage, starting for example with those of Hue et al. (2015, 2016, 2018). Given their extraordinary sensitivity, bandwidth, and spectral and spatial resolutions, future complementary ALMA and JWST observations will certainly help to shed light on the recent extraordinary
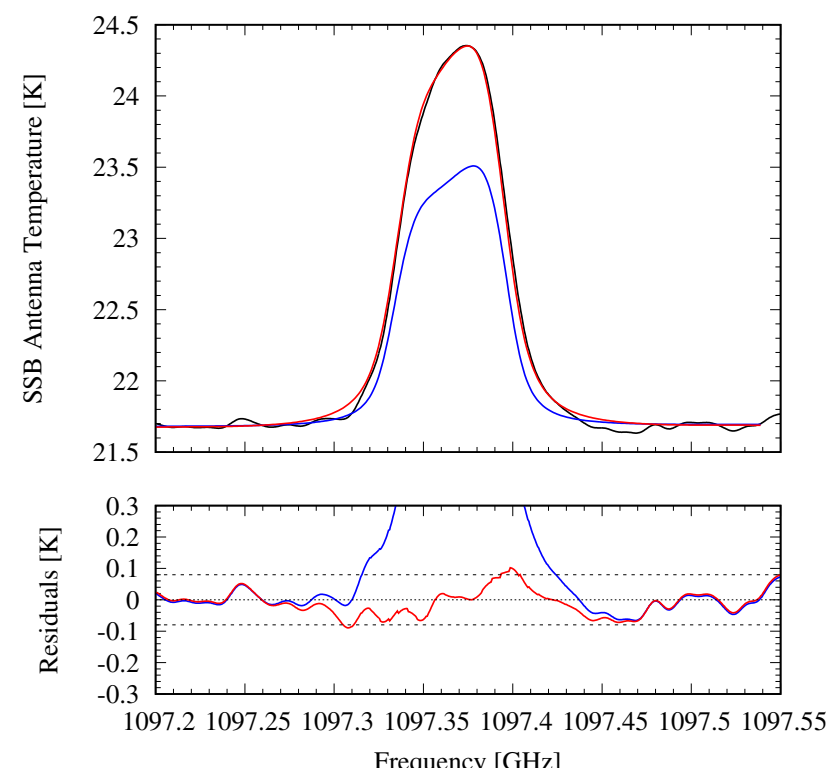

Fig. 17. Best fit to the HIFI data for a Gaussian distribution of $\mathrm{H}_{2} \mathrm{O}$ around the equator with $y_{\mathrm{eq}}=2.2 \times 10^{-7}$ and $\sigma=25^{\circ}$, after accounting for the effect of the temperature increases in $\mathrm{B} 1$ and $\mathrm{B} 2$ and a positive gradient for pressures higher than $p_{\text {gradient }}=0.1 \mathrm{mbar}$ and down to the condensation level with a slope $n=\log (y) / \log (p)=2$. The best-fit model to the PACS data from Fig. 16 is shown for comparison. Same layout as in Fig. 8 left.

influx of exogenic material from the inner ring system to Saturn's stratosphere, as captured by Cassini in its final orbits (Waite et al. 2018; Perry et al. 2018; Mitchell et al. 2018; Hsu et al. 2018). These future observations (Lellouch 2008; Norwood et al. 2016), as well as possibly direct in situ measurements (Mousis et al. 2014, 2016, 2018), will also help to improve our understanding of the outstanding and more general question of the origin of exogenic species in giant planet atmospheres.

Acknowledgements. This work was supported by the Programme National de Planétologie (PNP) of CNRS/INSU, co-funded by CNES. T.C. was supported by a CNES fellowship at the beginning of this work. Support for G.S.O. was provided by NASA through an award issued by the Jet Propulsion Laboratory, California Institute of Technology. PACS was developed by a consortium of institutes led by MPE (Germany) and including UVIE (Austria); KU Leuven, CSL, IMEC (Belgium); CEA, LAM (France); MPIA (Germany); INAF-IFSI/OAA/OAP/OAT, LENS, SISSA (Italy); IAC (Spain). This development has been supported by the funding agencies BMVIT (Austria), ESAPRODEX (Belgium), CEA/CNES (France), DLR (Germany), ASI/INAF (Italy), and CICYT/MCYT (Spain). HIFI was designed and built by a consortium of institutes and university departments from across Europe, Canada and the United States under the leadership of SRON Netherlands Institute for Space Research, Groningen, The Netherlands and with major contributions from Germany, France and the USA. Consortium members are: Canada: CSA, U.Waterloo; France: CESR, LAB, LERMA, IRAM; Germany: KOSMA, MPIfR, MPS; Ireland, NUI Maynooth; Italy: ASI, IFSI-INAF, Osservatorio Astrofisico di ArcetriINAF; Netherlands: SRON, TUD; Poland: CAMK, CBK; Spain: Observatorio Astronómico Nacional (IGN), Centro de Astrobiología (CSIC-INTA). Sweden: Chalmers University of Technology - MC2, RSS \& GARD; Onsala Space Observatory; Swedish National Space Board, Stockholm University - Stockholm Observatory; Switzerland: ETH Zurich, FHNW; USA: Caltech, JPL, NHSC. The Herschel spacecraft was designed, built, tested, and launched under a contract to ESA managed by the Herschel/Planck Project team by an industrial consortium under the overall responsibility of the prime contractor Thales Alenia Space (Cannes), and including Astrium (Friedrichshafen) responsible for the payload module and for system testing at spacecraft level, Thales Alenia Space (Turin) responsible for the service module, and Astrium (Toulouse) responsible for the telescope, with in excess of a hundred subcontractors. HCSS/HSpot/HIPE is a joint development (are joint developments) by the Herschel Science Ground Segment Consortium, consisting of ESA, the NASA Herschel Science Center, and the HIFI, PACS, and SPIRE consortia. 


\section{References}

Altobelli, N., Lopez-Paz, D., Pilorz, S., et al. 2014, Icarus, 238, 205 Bergin, E. A., Lellouch, E., Harwit, M., et al. 2000, ApJ, 539, L147 Bézard, B., Lellouch, E., Strobel, D., Maillard, J.-P., \& Drossart, P. 2002, Icarus, 159,95

Borysow, A., \& Frommhold, L. 1986, ApJ, 304, 849

Borysow, J., Trafton, L., Frommhold, L., \& Birnbaum, G. 1985, ApJ, 296, 644

Borysow, J., Frommhold, L., \& Birnbaum, G. 1988, ApJ, 326, 509

Brown, L. R., \& Plymate, C. 1996, J. Quant. Spectr. Rad. Transf., 56, 263

Burgdorf, M., Orton, G., van Cleve, J., Meadows, V., \& Houck, J. 2006, Icarus, 184,634

Cassidy, T. A., \& Johnson, R. E. 2010, Icarus, 209, 696

Cavalié, T., Billebaud, F., Biver, N., et al. 2008a, Planet. Space Sci., 56, 1573

Cavalié, T., Billebaud, F., Fouchet, T., et al. 2008b, A\&A, 484, 555

Cavalié, T., Billebaud, F., Dobrijevic, M., et al. 2009, Icarus, 203, 531

Cavalié, T., Hartogh, P., Billebaud, F., et al. 2010, A\&A, 510, A88

Cavalié, T., Biver, N., Hartogh, P., et al. 2012, Planet. Space Sci., 61, 3

Cavalié, T., Feuchtgruber, H., Lellouch, E., et al. 2013, A\&A, 553, A21

Cavalié, T., Moreno, R., Lellouch, E., et al. 2014, A\&A, 562, A33

Cavalié, T., Venot, O., Selsis, F., et al. 2017, Icarus, 291, 1

Connerney, J. E. P. 1986, Geophys. Res. Lett., 13, 773

Connerney, J. E. P., \& Waite, J. H. 1984, Nature, 312, 136

Conrath, B. J., \& Gautier, D. 2000, Icarus, 144, 124

Coustenis, A., Salama, A., Lellouch, E., et al. 1998, A\&A, 336, L85

Davis, G. R., Griffin, M. J., Naylor, D. A., et al. 1996, A\&A, 315, L393

de Graauw, T., Feuchtgruber, H., Bezard, B., et al. 1997, A\&A, 321, L13

de Graauw, T., Helmich, F. P., Phillips, T. G., et al. 2010, A\&A, 518, L6

Dick, M. J., Drouin, B. J., \& Pearson, J. C. 2009, J. Quant. Spectr. Rad. Transf., 110,619

Dobrijevic, M., Hébrard, E., Loison, J. C., \& Hickson, K. M. 2014, Icarus, 228, 324

Dunn, D. E., de Pater, I., Wright, M., Hogerheijde, M. R., \& Molnar, L. A. 2005, AJ, 129, 1109

Feuchtgruber, H., Lellouch, E., de Graauw, T., et al. 1997, Nature, 389, 159

Fischer, G., Kurth, W. S., Gurnett, D. A., et al. 2011, Nature, 475, 75

Flandes, A., Spilker, L., Morishima, R., et al. 2010, Planet. Space Sci., 58, 1758

Fletcher, L. N., Irwin, P. G. J., Teanby, N. A., et al. 2007, Icarus, 188, 72

Fletcher, L. N., Orton, G. S., Teanby, N. A., Irwin, P. G. J., \& Bjoraker, G. L. 2009a, Icarus, 199, 351

Fletcher, L. N., Orton, G. S., Teanby, N. A., \& Irwin, P. G. J. 2009b, Icarus, 202, 543

Fletcher, L. N., Hesman, B. E., Irwin, P. G. J., et al. 2011, Science, 332, 1413

Fletcher, L. N., Swinyard, B., Salji, C., et al. 2012, A\&A, 539, A44

Fletcher, L. N., Guerlet, S., Orton, G. S., et al. 2017, Nat. Astron., 1, 765

Fletcher, L. N., Gustafsson, M., \& Orton, G. S. 2018a, ApJS, 235, 24

Fletcher, L. N., Orton, G. S., Sinclair, J. A., et al. 2018b, Nat. Commun., 9, 3564

Fouchet, T., Guerlet, S., Strobel, D. F., et al. 2008, Nature, 453, 200

Fray, N., \& Schmitt, B. 2009, Planet. Space Sci., 57, 2053

Friedson, A. J., \& Moses, J. I. 2011, AGU Fall Meeting Abstracts, P13C

Gautier, D., Hersant, F., Mousis, O., \& Lunine, J. I. 2001, ApJ, 550, L227

Guerlet, S., Fouchet, T., Bézard, B., Simon-Miller, A. A., \& Michael Flasar, F. 2009, Icarus, 203, 214

Guerlet, S., Fouchet, T., Bézard, B., et al. 2010, Icarus, 209, 682

Guerlet, S., Fouchet, T., Bézard, B., Flasar, F. M., \& Simon-Miller, A. A. 2011, Geophys. Res. Lett., 38, L09201

Guerlet, S., Spiga, A., Sylvestre, M., et al. 2014, Icarus, 238, 110

Hansen, C. J., Esposito, L., Stewart, A. I. F., et al. 2006, Science, 311, 1422

Hartogh, P., Lellouch, E., Crovisier, J., et al. 2009, Planet. Space Sci., 57, 1596

Hartogh, P., Lellouch, E., Moreno, R., et al. 2011, A\&A, 532, L2

Hedman, M. M., \& Showalter, M. R. 2016, Icarus, 279, 155

Hersant, F., Gautier, D., \& Lunine, J. I. 2004, Planet. Space Sci., 52, 623

Hickson, K. M., Loison, J. C., Cavalié, T., Hébrard, E., \& Dobrijevic, M. 2014, A\&A, 572, A58

Higgins, R., Teyssier, D., Borys, C., et al. 2014, Exp. Astron., 37, 433

Hsu, H.-W., Schmidt, J., Kempf, S., et al. 2018, Science, 362, 3185

Hue, V., Cavalié, T., Dobrijevic, M., Hersant, F., \& Greathouse, T. K. 2015, Icarus, 257, 163

Hue, V., Greathouse, T. K., Cavalié, T., Dobrijevic, M., \& Hersant, F. 2016, Icarus, 267, 334

Hue, V., Hersant, F., Cavalié, T., Dobrijevic, M., \& Sinclair, J. A. 2018, Icarus, 307, 106

Johnson, R. E., Luhmann, J. G., Tokar, R. L., et al. 2006, Icarus, 180, 393

Jurac, S., \& Richardson, J. D. 2007, Geophys. Res. Lett., 34, L08102
Landgraf, M., Liou, J.-C., Zook, H. A., \& Grün, E. 2002, AJ, 123, 2857

Lara, L. M., Lellouch, E., González, M., Moreno, R., \& Rengel, M. 2014, A\&A, 566, A143

Larson, H. P., Fink, U., Treffers, R., \& Gautier, III, T. N. 1975, ApJ, 197, L137

Lellouch, E. 2008, Ap\&SS, 313, 175

Lellouch, E., Paubert, G., Moreno, R., et al. 1995, Nature, 373, 592

Lellouch, E., Bézard, B., Moses, J. I., et al. 2002, Icarus, 159, 112

Lellouch, E., Moreno, R., \& Paubert, G. 2005, A\&A, 430, L37

Lellouch, E., Bézard, B., Strobel, D. F., et al. 2006, Icarus, 184, 478

Lellouch, E., Hartogh, P., Feuchtgruber, H., et al. 2010, A\&A, 518, L152

Levy, A., Lacome, N., \& Tarrago, G. 1993, J. Mol. Spectr., 157, 172

Levy, A., Lacome, N., \& Tarrago, G. 1994, J. Mol. Spectr., 166, 20

Lindal, G. F., Sweetnam, D. N., \& Eshleman, V. R. 1985, AJ, 90, 1136

Lodders, K., \& Fegley, Jr. B. 1994, Icarus, 112, 368

Lomb, N. R. 1976, Ap\&SS, 39, 447

Luszcz-Cook, S. H., \& de Pater, I. 2013, Icarus, 222, 379

Mandt, K. E., Mousis, O., Marty, B., et al. 2015, Space Sci. Rev., 197, 297

Marten, A., Gautier, D., Owen, T., et al. 1993, ApJ, 406, 285

Marten, A., Matthews, H. E., Owen, T., et al. 2005, A\&A, 429, 1097

Mitchell, D. G., Perry, M. E., Hamilton, D. C., et al. 2018, Science, 362, aat2236

Moore, L., Mueller-Wodarg, I., Galand, M., Kliore, A., \& Mendillo, M. 2010, J. Geophys. Res. Space Phys., 115, A11317

Moore, L., O’Donoghue, J., Müller-Wodarg, I., Galand, M., \& Mendillo, M. 2015, Icarus, 245, 355

Moreno, R., Marten, A., Matthews, H. E., \& Biraud, Y. 2003, Planet. Space Sci., 51,591

Moreno, R., Lellouch, E., Lara, L. M., et al. 2012, Icarus, 221, 753

Moreno, R., Lellouch, E., Cavalié, T., \& Moullet, A. 2017, A\&A, 608, L5

Moses, J. I., \& Poppe, A. R. 2017, Icarus, 297, 33

Moses, J. I., Lellouch, E., Bézard, B., et al. 2000, Icarus, 145, 166

Moses, J. I., Fouchet, T., Bézard, B., et al. 2005, J. Geophys. Res. Planets, 110, E08001

Mousis, O., Fletcher, L. N., Lebreton, J.-P., et al. 2014, Planet. Space Sci., 104 29

Mousis, O., Atkinson, D. H., Spilker, T., et al. 2016, Planet. Space Sci., 130, 80

Mousis, O., Atkinson, D. H., Cavalié, T., et al. 2018, Planet. Space Sci., 155, 12

Noll, K. S., Knacke, R. F., Geballe, T. R., \& Tokunaga, A. T. 1986, ApJ, 309, L91

Norwood, J., Moses, J., Fletcher, L. N., et al. 2016, PASP, 128, 018005

O’Donoghue, J., Moore, L., Connerney, J. E. P., et al. 2017, Geophys. Res. Lett., 44,11

Ollivier, J. L., Dobrijévic, M., \& Parisot, J. P. 2000, Planet. Space Sci., 48, 699

Orton, G. S., Gustafsson, M., Burgdorf, M., \& Meadows, V. 2007, Icarus, 189, 544

Orton, G. S., Yanamandra-Fisher, P. A., Fisher, B. M., et al. 2008, Nature, 453, 196

Ott, S. 2010, PASP, 434, 139

Owen, T., \& Encrenaz, T. 2003, Space Sci. Rev., 106, 121

Perry, M. E., Waite Jr. J. H., Mitchell, D. G., et al. 2018, Geophys. Res. Lett., 45, 10,093

Pickett, H. M., Poynter, R. L., Cohen, E. A., et al. 1998, J. Quant. Spectr. Rad. Transf., 60, 883

Pilbratt, G. L., Riedinger, J. R., Passvogel, T., et al. 2010, A\&A, 518, L1

Poglitsch, A., Waelkens, C., Geis, N., et al. 2010, A\&A, 518, L2

Poppe, A. R. 2016, Icarus, 264, 369

Porco, C. C., Helfenstein, P., Thomas, P. C., et al. 2006, Science, 311, 1393

Prangé, R., Fouchet, T., Courtin, R., Connerney, J. E. P., \& McConnell, J. C. 2006, Icarus, 180, 379

Rengel, M., Sagawa, H., Hartogh, P., et al. 2014, A\&A, 561, A4

Rezac, L., de Val-Borro, M., Hartogh, P., et al. 2014, A\&A, 563, A4

Roelfsema, P. R., Helmich, F. P., Teyssier, D., et al. 2012, A\&A, 537, A17

Samuelson, R. E., Maguire, W. C., Hanel, R. A., et al. 1983, J. Geophys. Res., 88,8709

Sánchez-Lavega, A., del Río-Gaztelurrutia, T., Hueso, R., et al. 2011, Nature, 475,71

Sinclair, J. A., Irwin, P. G. J., Fletcher, L. N., et al. 2013, Icarus, 225, 257

Spilker, L., Ferrari, C., Cuzzi, J. N., et al. 2003, Planet. Space Sci., 51, 929

Spilker, L. J., Pilorz, S. H., Edgington, S. G., et al. 2005, Earth Moon Planets, 96,149

Strobel, D. F., \& Yung, Y. L. 1979, Icarus, 37, 256

van der Tak, F., de Pater, I., Silva, A., \& Millan, R. 1999, Icarus, 142, 125

Waite, J. H., Combi, M. R., Ip, W.-H., et al. 2006, Science, 311, 1419

Waite, J. H., Perryman, R. S., Perry, M. E., et al. 2018, Science, 362, 51

Wang, D., Gierasch, P. J., Lunine, J. I., \& Mousis, O. 2015, Icarus, 250, 154

Weisstein, E. W., \& Serabyn, E. 1996, Icarus, 123, 23 
T. Cavalié et al.: Herschel map of Saturn's stratospheric water, delivered by the plumes of Enceladus

\section{Appendix A: Herschel-PACS raw spectra}

The 225 raw spectra obtained with Herschel-PACS on the $3 \times 3$ positions of the raster map are presented in Fig. A.1.
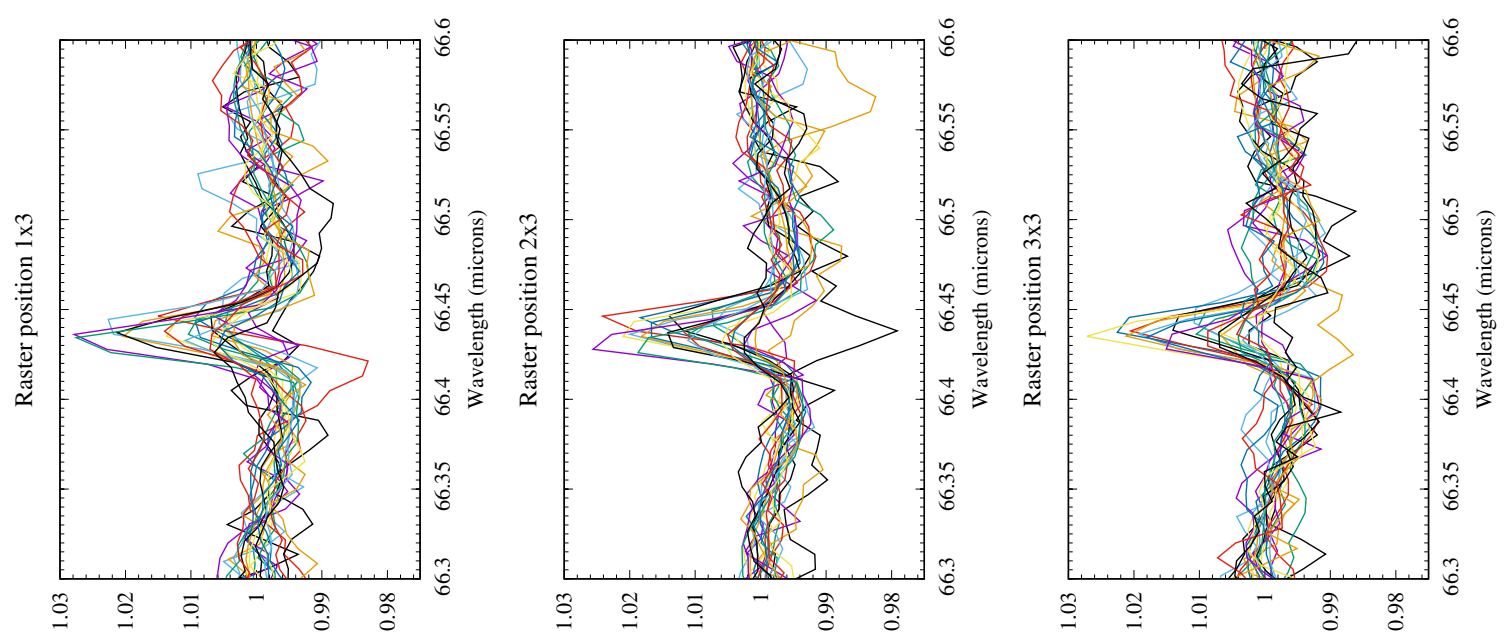

ว/I

ग/[

ग/I
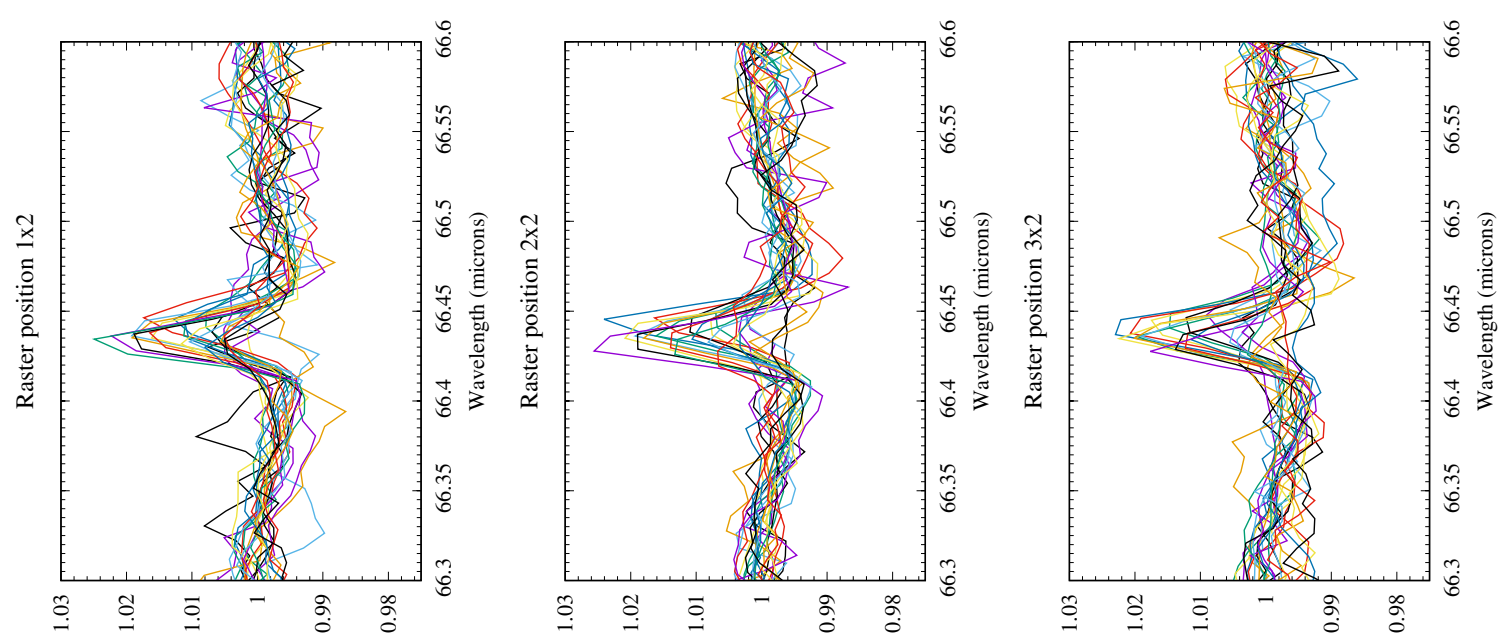

ग/I

\%/I

\%/
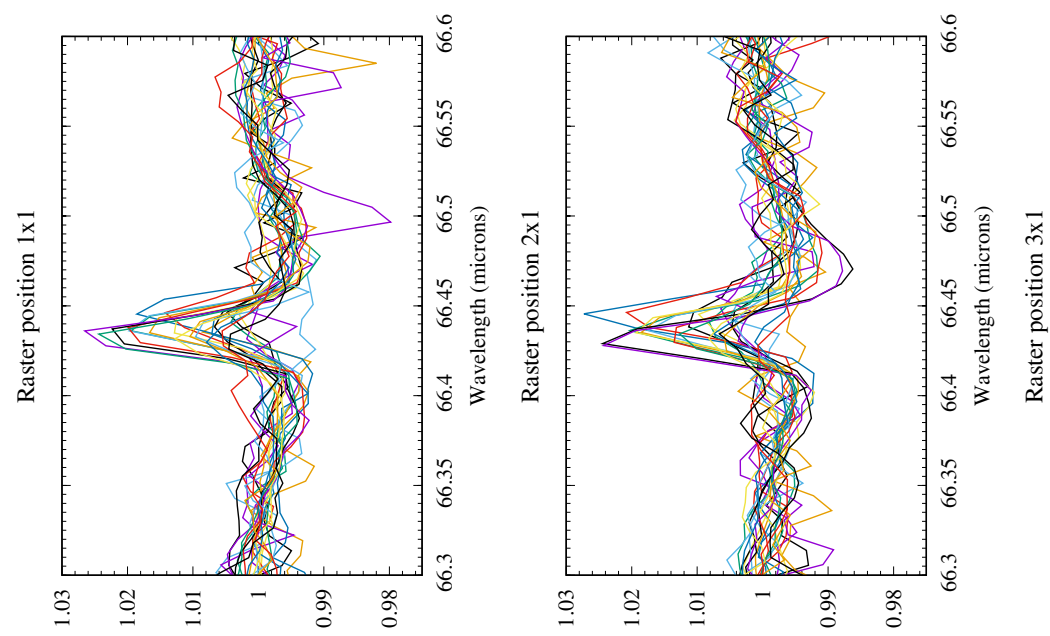

o/I

\%/I

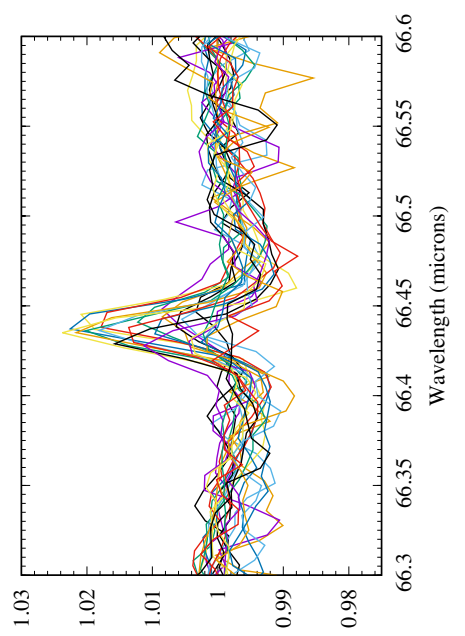

ग/I

Fig. A.1. Two hundred and twenty-five raw spectra obtained with the PACS $5 \times 5$ detector array on all of the $3 \times 3$ raster map positions on Saturn at $66.44 \mu \mathrm{m}$. The 25 spectra recorded by the detector array are plotted for each of the 9 raster map positions. The $\mathrm{H}_{2} \mathrm{O}$ line is detected in all spectra corresponding to pointings on the planetary disk or close to the planetary limb. Spectra without an $\mathrm{H}_{2} \mathrm{O}$ line correspond to pointings far off the planetary limb. Before fitting the lines with a Gaussian to compute their area, a polynomial baseline was removed from these spectra. The line peak $\mathrm{S} / \mathrm{N}$ around the limb is $\sim 15-45$. 